\title{
Of Local Operations and Physical Wires
}

\author{
Dario Egloff, ${ }^{1, *}$ Juan M. Matera, ${ }^{2}$ Thomas Theurer, ${ }^{1}$ and Martin B. Plenio ${ }^{1}$ \\ ${ }^{1}$ Institute of Theoretical Physics and IQST, Universität Ulm, Albert-Einstein-Allee 11, \\ D-89069 Ulm, Germany \\ ${ }^{2}$ IFLP-CONICET, Departamento de Física, Facultad de Ciencias Exactas, \\ Universidad Nacional de La Plata, C.C. 67, La Plata 1900, Argentina
}

(Received 20 February 2018; revised manuscript received 25 April 2018; published 6 July 2018)

\begin{abstract}
In this work (multipartite) entanglement, discord, and coherence are unified as different aspects of a single underlying resource theory defined through simple and operationally meaningful elemental operations. This is achieved by revisiting the resource theory defining entanglement, local operations, and classical communication (LOCC), placing the focus on the underlying quantum nature of the communication channels. Taking the natural elemental operations in the resulting generalization of LOCC yields a resource theory that singles out coherence in the wire connecting the spatially separated systems as an operationally useful resource. The approach naturally allows us to consider a reduced setting as well, namely, the one with only the wire connected to a single quantum system, which leads to discordlike resources. The general form of free operations in this latter setting is derived and presented as a closed form. We discuss in what sense the present approach defines a resource theory of quantum discord and in which situations such an interpretation is sound-and why in general discord is not a resource. This unified and operationally meaningful approach makes transparent many features of entanglement that in LOCC might seem surprising, such as the possibility to use a particle to entangle two parties, without it ever being entangled with either of them, or that there exist different forms of multipartite entanglement.
\end{abstract}

DOI: 10.1103/PhysRevX.8.031005

Subject Areas: Quantum Physics,

Quantum Information

\section{INTRODUCTION}

One of the oldest questions in the field of quantum mechanics is how quantum states differ from classical states. While certainly it is hard to compare frameworks that are so fundamentally different in nature, the main qualitative difference on a formal level is that quantum mechanics deals with probability amplitudes instead of probabilities. As a consequence, one of the main predictions of quantum mechanics is that physical systems can exhibit coherent superpositions of those states associated to sharply defined values of the observables [1], which can, for instance, be observed in interference experiments. It is mainly this difference that prevents quantum theory from being explicable by a deterministic hidden variable model with variables that are local [2-6] or noncontextual [7-9]. The question then becomes how one can understand this difference in detail and, furthermore, how one can quantify

*dario.egloff@uni-ulm.de

Published by the American Physical Society under the terms of the Creative Commons Attribution 4.0 International license. Further distribution of this work must maintain attribution to the author(s) and the published article's title, journal citation, and DOI. it. This is relevant in its own right and also to give solid foundations to the debates about how quantum mechanical observed coherences in biological systems [10] are, or to objectively compare different platforms for quantum computers by measuring how much more resources they provide than their classical counterparts.

A particularly transparent approach is given by the theory of local operations and classical communication (LOCC) (see Refs. [11,12] for reviews), which incorporates the idea of the impossibility of creating nonlocal superpositions (entanglement) if one has two distant parties that can only communicate classically, akin to Bell's argument for the nonclassicality of quantum physics [3] (see Sec. III).

However, entanglement is not the only nonclassical feature of quantum mechanics. There are other forms of superposition that can also give advantages over classical states. An instance in which this becomes apparent is the protocol of deterministic quantum computing with one qubit (DQC1) that can outperform any known classical algorithm, even if no bipartite entanglement is present [13-17]. It was argued that a property denoted as discord [18-21] would be the resource responsible for this operational advantage. While discord is an interesting measure, it is hard to argue that it is a resource of nonclassicality, since it can be created already by mixing discord-free states (in 
fact, product states) and mixing states amounts to forgetting information - a task that is easily accomplished classically [18]. Some advances in understanding the resources in DQC1 have recently been made using a different form of nonclassicality, called coherence, which is exhibited by superpositions of states in a fixed orthonormal basis, whose elements and their statistical mixtures are the incoherent states [22-25]. Based on this theory, complementing recently studied connections between entanglement and coherence [26-39], it was shown that the precision of the DQC1 protocol is a function of the coherence of the qubit one uses as a control and that any state with some property called basis-dependent discord [39-43] is a resource in this setting $[42,43]$.

The standard (basis-independent) discord is recovered when basis-dependent discord is minimized over different bases [43]. Ironically, in the theories used in these papers, which explore more generic nonclassical resources than entanglement, coherent states and entangled states are interconvertible resources and optimal instances of basisdependent discord. Even more generally, there is an operation that maps any superposition of a given set of linearly independent states (not necessarily orthogonal) to entangled states and nonsuperposition states to separable states [44-46]. This means that, while entanglement does not seem to be the most generic nonclassical resource, as it does not easily encompass all forms of superposition that seem useful for quantum tasks, the resources that enable to produce entanglement might well be. Seemingly, the ability to do something truly quantum entails the possibility to produce entanglement. It is this "universal character" [47] of entanglement that motivates the present paper.

\section{OUTLINE AND SUMMARY OF THE MAIN RESULTS}

The aim of this paper is to unify different types of nonclassicality: entanglement, discord, basis-dependent discord, and coherence.

The starting point is the resource theory with the clearest operational interpretation so far, namely, that arising from the constraint to LOCC [48]. In that framework, one has two distant parties (Alice and Bob) that can implement arbitrary local operations, but can only communicate classically. In LOCC, this classical communication is treated only abstractly, even though clearly it happens through a physical system, which we call the wire. In the resource theory that we introduce, local operations and physical wires (LOP), the wire is explicitly included in the description (see Fig. 1). That is, we treat the wire needed for classical communication as a quantum system. To get back the same effective free operations as in LOCC, we need to define free operations on the wire that correspond to classical communication. We define them as encoding some classical data into a fixed basis, relabeling the basis states, forwarding a part of the system to Alice or Bob, and

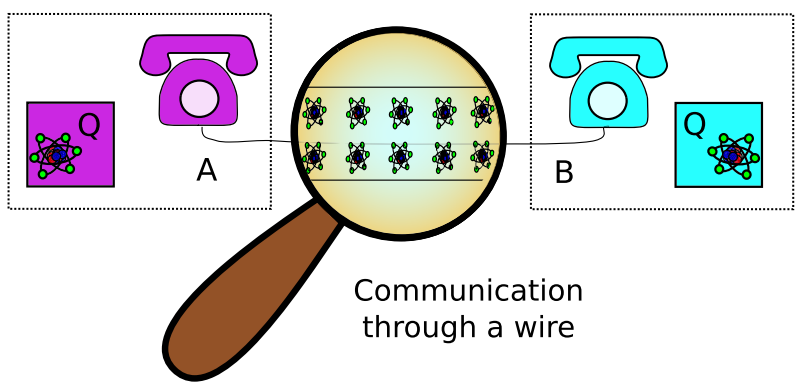

FIG. 1. Wires for classical communication are quantum systems.

iterations of these (see Fig. 2). This framework is presented in Sec. III A. What we gain is a clear interpretation of what is classical from the point of view of the wire. Indeed, we can look at the wire connected to only one system, recovering a setting in which basis-dependent discord becomes the resource. We also look at the effective theory considering the wire alone, which defines an operationally motivated theory of coherence. Results for these two last cases are discussed in the remainder of Sec. III.

As is usually the case in resource theories, free states are those that can be prepared by free operations; in the setting with one wire connected with one quantum system (say, Alice), the free states are classical-quantum [meaning $\sum_{m} p_{m}|m\rangle\left\langle\left. m\right|_{W} \otimes \sigma_{m Q}\right.$, where the indices just denote the wire $(W)$, respectively, and the quantum system of Alice $(Q)$ $[26,42]]$. In this setting, all nonfree states can be used as resources to promote an operation that had a clear classical interpretation on the subsystem of the wire, to an arbitrary quantum operation. That is, having a sufficiently large number of subsystems that are not classical-quantum, one can do any quantum operation on an additional subsystem only using free operations. The nonfree operations are thus made possible by consuming nonfree states, that is, mapping them to free states. This result follows from Theorem 5, where the usefulness of maximally coherent states is shown, together with the result that one can distillate maximally coherent states from any other states that are not free, as discussed in Appendix E. More details on this can be found in the last two paragraphs of Sec. III B.

In Sec. III B we also present the (technical, but useful) result that there is an explicitly finite representation of the free operations (Proposition 3 and Fig. 3). This allows us to prove an alternative interpretation of the free operations (Proposition 4), which clarifies the connection with other approaches to coherence theory [22-24,39-41,49-51] and basis-dependent quantum discord [39-43] (Proposition 6); see Fig. 4 and Appendix B. The main result here is that the theory is very similar - though not equivalent - to the more abstract theories of coherence or basis-dependent discord, defined in Refs. [23,39,43].

In similar settings, the usual argument to call one basis of the wire "incoherent" (or-sloppily_-"classical") is that 
one assumes some dephasing, imposed by the natural evolution of the system. While this interpretation is still meaningful in our setting, it is not necessary. It can make perfect sense to have a more subjective choice of the incoherent basis. For instance, the incoherent basis could just be the one in which Alice chooses to encode her information, or it could also happen that there is a natural dephasing to an unknown basis. In these latter cases, it can seem natural to optimize over the possible incoherent bases, giving a natural interpretation of discord (also see Refs. [39-43]). This is discussed in detail in Sec. III C.

Entanglement theory is discussed in Sec. IV. By construction, LOP entails LOCC; and they are equivalent, if one only considers the effective theory of LOP on the different parties without wire (Remark 9). Even more, coherence on the wire in the bipartite LOP setting is exactly as useful as the correspondent maximally correlated state for bipartite LOCC (see Theorem 7 for the usefulness of the resources for operations and its Corollary 8 for state transformations). One might expect this from a similar relationship between different forms of quantum cryptography (see, e.g., Refs. [52,53]). This setting thus explains the recently studied relation between these different resources [26-43] as the interplay of different facets of the same resource theory.

As noted in the abstract, it is possible to entangle two distant parties Alice and Bob, by sending one quantum particle from Alice to Bob that is never entangled with Alice or Bob [54]. Since in our setting sending a particle can be described explicitly, we can analyze the resources involved. This is also done in Sec. IV. As the argument is straightforward, we reiterate it here. Since entanglement is a resource in LOP, and forwarding a particle from the wire to Bob is a free operation, there necessarily needs to have been some resource on the subsystem of Alice and the wire, i.e., some basis-dependent discord. Note, however, that this statement needs to be true independently of the basis one considers incoherent for the wire, which means that the state necessarily has nonzero quantum discord (we discuss in Sec. III C how this can be reconciled with the fact that quantum discord is not a resource).

Finally we look at the multipartite case, where one has different possible natural generalizations of multipartite LOCC, depending on how one connects the parties by wires (see Figs. 5 and 6). Here, we could not prove a one-to-one correspondence between the values of coherence on the wires in LOP and multipartite entanglement. However, the coherence cost of producing entangled states still gives a bound on what state conversions are possible under LOCC, even in the multipartite case (Theorem 10). Using this, we showed that, for three parties, two different wiring schemes reverse the coherence cost of producing the $G H Z$ and the $W$ state. This suggests that different wiring schemes might be connected to different classes of entanglement (see, e.g., Refs. [55-60]). All proofs are in the Appendix and linked in to the propositions.

\section{LOCAL OPERATIONS AND PHYSICAL WIRES}

\section{A. Setting}

As explained in the Introduction, we are aiming at understanding and quantifying nonclassicality better. The tool of choice to develop this understanding is resource theories, since they provide a systematic guide for analyzing situations where one wants to find and/or quantify the properties that can be useful for some tasks.

Abstractly, and glossing over details, to get a resource theory, one puts a meaningful, but artificial, restriction on what operations are allowed (free), within a given framework. The restriction should be chosen such that the connection to the property one wants to focus on is as clear as possible (which does not need to coincide with the distinction between "easy" and "hard"). There may be some preparations that are free operations and, accordingly, some states that are free. Since the states that are not free cannot be prepared by free operations, in some cases, they might help to do an operation that otherwise would not be free; these are the resource states. There are more useful states and less useful states (if from one state one can reach another one with free operations, the first is more useful, since it can be used for anything the second can be), imposing a partial order on the states. A measure for the resource can, therefore, only be meaningful if it is monotonous under the free operations, restricting strongly possible candidates.

One of the most successful resource theories in quantum information, and the starting point of our considerations, is the theory of LOCC. This theory aims to capture the idea of Einstein, Podolsky and Rosen (EPR) and Bell that, in classical physics, it is not possible to reproduce the effect of having nonlocal superpositions of states [2,3]. LOCC can be described by its elemental operations, consisting of arbitrary local quantum operations on one system, postselection, and classical broadcasting of the result. Any LOCC operation is a concatenation of such operations (potentially depending on the results of the previous ones). Here, we want to treat the broadcasting as an operation using a physical wire, instead of how it is usually done as an implicit exchange of classical information. The broadcasting is then simply given by forwarding the state of the wire as an ancilla to the party in question.

In this way, it makes sense to talk about the state of the communication channel as a quantum state. The standard LOCC theory is recovered by assuming that the state of the wire is forced to be incoherent, i.e., $\rho_{\text {wires }}=\sum_{i} p(i)|i\rangle\langle i|$, and in a product state with both parties, meaning that the probability distribution is encoded in the diagonals. Note that, while the basis one uses to encode a probability distribution in the wire is in principle arbitrary, one needs to fix it in advance. To be precise, while it may change in time, this change must not depend on the measurement outcomes of the protocol; it must be defined at the beginning of the protocol. Henceforth, we will call this basis incoherent and 
denote it by $\mathcal{Z}$. With this definition, it also makes sense to allow some classical processing in the wire as a free operation on this extended theory, that is, permutations of these basis states. As an example of why it is important to fix the basis, and, therefore, which states are incoherent, in advance, to get a fair description of the role coherence plays, let us consider the BB84 protocol [61].

The goal of BB84 is to distribute random keys in a safe way. This is achieved by sending qubits through a quantum channel where each of them is encoded by Alice in one of two possible noncommuting bases, depending on the outcome of a random measurement. On the other side, Bob measures the qubit in a random basis. After repeating this many times, Alice and Bob publicly compare the bases chosen and keep only the data of the measurements that were done in the same bases. They can then compare a fraction of the remaining data to be certain (in the asymptotic limit) that nobody interfered. A naive approach to describe this protocol is that, as in each round the state of the qubit sent is diagonal in some basis, then the full protocol is classical. Why does this algorithm beat classical key distribution? The crucial departure from classical physics of this protocol is the random choice of bases, and the security of the algorithm relies on the fact that the choice of the basis in which the qubits are diagonal is unknown to a possible eavesdropper Eve. Therefore, to analyze the security of this protocol, we need to take the perspective of Eve. For her, whatever basis she assumes to be incoherent, there will be some states sent by Alice that will be coherent, given a long enough sequence. This is the case, because she does not know the outcomes of Alice's measurements that defined the encoding bases. For this reason, a fair description of what Eve can do needs to assume that she chooses the basis before knowing the basis in which Alice encodes the state.

As noted in the Introduction, we only need one quantum system $Q$, together with a wire $W$, to make sense of this theory. We start by describing this case in some detail, before coming back to the case with multiple parties $Q=$ $\otimes_{i} Q^{i}$ and wires $W=\otimes_{j} W^{j}$. For simplicity, to change the phases of the basis states is also assumed to be a free operation-but, as shown below, this does not significantly alter the theory. We assume that both $Q$ and $W$ are finitedimensional systems, but do not keep their dimensions fixed (see footnote [62] for more details). The free operations in this case consist of iteratively applying the following elemental Kraus operations [which should form a completely positive and trace preserving (CPTP) map on $W \otimes Q$, that is, $\sum_{\alpha} K^{\alpha \dagger} K^{\alpha}=\mathbb{1}$ ] and postselecting (for a neater notation, we only write out the spaces on which the Kraus operations act nontrivially and, on all others, the identity operation is assumed):

(1) Permutations: Permutations $\sigma$ of basis states in the basis $\mathcal{Z}$ on the wire $W, \sum_{i}|\sigma(i)\rangle\left\langle\left. i\right|_{W}\right.$.
(2) Phases: Diagonal unitary evolutions in the basis $\mathcal{Z}$ on $W, \sum_{j} e^{i \phi(j)}|j\rangle\left\langle\left. j\right|_{W}\right.$.

(3) Observed quantum operations: Any generalized measurements on $Q$, encoding its outcomes as an incoherent state of an ancillary subsystem $W_{a}$ of $W$, $|\alpha\rangle_{W_{a}} \otimes F_{Q}^{\alpha}$, for Kraus operators $F_{Q}^{\alpha}$ acting on $Q$.

(4) Classical to quantum forwarding: Transfer a subsystem $W_{s}$ of $W$ to a subsystem $Q_{t}$ of $Q: \mathbb{1}_{Q_{t} \leftarrow W_{s}}=$ $\sum_{j}|j\rangle_{Q_{t}}\left\langle\left. j\right|_{W_{s}}=\sum_{j}\left\langle\left. j\right|_{W_{s}} \otimes \mid j\right\rangle_{Q_{t}}[62]\right.$.

For simplicity, here, we assume the incoherent basis $\mathcal{Z}$ to be the same for all times, but one can easily get the more general setting by replacing the free operations $K^{\alpha}$ by $\left[U_{W}\left(t, t_{0}\right) \otimes \mathbb{1}_{Q}\right] K^{\alpha}$, where $U_{W}\left(t, t_{0}\right)$ defines the (necessarily predefined; see above) change of basis for the time step $t_{0} \rightarrow t$. Note that this includes replacing the identity by $\left[U_{W}\left(t, t_{0}\right) \otimes \mathbb{1}_{Q}\right]$ and that the identity operation itself is not a free operation any more. Sticking to this rule, the results stay unchanged, because concatenating free operations on $t_{0}, t_{1}$ and $t_{1}, t_{2}$ yields free operations on $t_{0}, t_{2}$. This is relevant if one wants to change the basis one calls classical in time, as is usually the case in settings where discord is thought to be a meaningful measure. We will come back to this in Sec. III C.

Note also that we treat encoding and decoding asymmetrically. The rationale we employ here is that it is hard to encode quantum information in a wire, but if coherence is provided and it is possible to sustain, transport, and control it, you may very well also be able to use it. That is why we only allow encoding classical information in the wire, while any state can be retrieved. We call the set of free operations in this theory of local operations and physical wires $\operatorname{LOP}(W \rightsquigarrow Q)=$ LOP, where here we use the symbols $\leftarrow$ and $\rightarrow$ for classical encoding, while $w$ and $\rightsquigarrow$ are used for transferring a quantum system. Items 3 and 4 are depicted in Fig. 2. We conclude this subsection by defining LOP with the promised proposition, which states that the phases

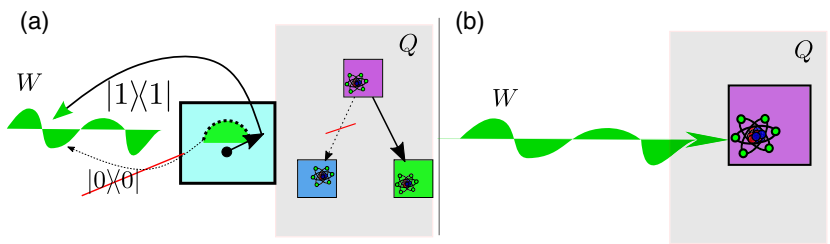

FIG. 2. The picture depicts items 3 and 4 of the elemental operations defining LOP. Item 3, depicted in panel (a), means that to do operations on the quantum state, measure what happens, and use the incoherent basis of the wire to encode the observation is considered to be a classical operation on the wire and, therefore, free. Item 4, depicted in panel (b), means that the wire is effectively treated as a quantum system. This means that it can interact with the quantum system in a quantum way. However, if the wire is an extended object, it might be hard to change its state in a controlled way by this interaction. Therefore it is considered a free operation to forward the state of a subsystem of the wire to the quantum side, but not to alter the state of the wire in a quantum way. 
are not really relevant (remember that all proofs can be found in the appendix):

Proposition 1. Any operation in LOP can be done with an arbitrarily high probability of success by a combination of permutations, observed quantum operations, and classical to quantum forwarding.

\section{B. Basis-dependent discord and coherence}

In this subsection, we give alternative formulations of the resource theory of LOP, defined in the last section, deepening its understanding. These formulations will prove useful to connect the theory to other approaches to basisdependent discord and coherence. These results are summarized in Fig. 4. It is easy to see that the set of free states that can be prepared (probabilistically or not) by LOP is given by the so-called incoherent-quantum states, $C Q_{\mathcal{Z}}^{(n)}=$ $\left.\left\{\rho\left|\rho=\sum_{m} p_{m}\right| m\right\rangle\left\langle\left. m\right|_{W} \otimes \sigma_{m Q}, \mid m\right\rangle \in \mathcal{Z}\right\}[26,42]$. Therefore, LOP is a subset of the incoherent-quantum operations (IQO) that is given by any operations that map incoherentquantum states to incoherent-quantum states, even after postselection [42]. It is not so easy to see how strict the inclusion is. We will discuss this question later in Proposition 6, once we have gathered more insight into the theory.

To get a clearer idea of the operations at hand, we will need to find a more compact form of the operations belonging to LOP. To this end and of independent interest, it is helpful to know bijections that are elements of LOP. Knowing bijections in a resource theory is useful because they define equivalence classes on states: All states that can be reached by bijections can be freely interconverted, making them equivalent resources (as for any task one state can be used for, any state that can be mapped into it is at least as useful). A trivial bijection in LOP is given by any unitary on $Q$, which means that any measure of the theory necessarily has to be invariant under the basis changes of $Q$. The following lemma establishes such a bijection between the states on the wire $W$ and maximally correlated states on $W \otimes Q$ (also see [26,31]):

Lemma 2. The operator $B: W \rightarrow W \otimes Q, \quad B=$ $\sum_{i}|i\rangle\left\langle\left. i\right|_{W} \otimes \mid i\right\rangle_{Q}$ defines an injection and defining $\mathcal{B}[\rho]=$ $\left(B \rho B^{\dagger}\right), \mathcal{B} \in$ LOP. Conversely there exists a map $\mathcal{B}^{-1}$ : $W \otimes Q \rightarrow W$, with $\mathcal{B}^{-1} \circ \mathcal{B}=\mathbb{1}_{W}$ and $\mathcal{B}^{-1} \in$ LOP.

We are now ready to give an equivalent characterization of the operations in LOP, which has the advantage of being an explicitly finite concatenation of maps of one fixed form. On one hand, this might help to find a minimal number of necessary Kraus operators [63]; on the other, a priori it is not obvious that such a simplification exists, since for LOCC, which is a very similar theory, the number of rounds needed for a transformation is unbounded [64]. The proposition states that one can write any element of LOP as a concatenation of $N$ LOP maps (and $N$ is bounded by the Hilbert space dimension of the wire) that are composed of
Kraus operators $K_{j}^{\alpha_{j}}$ having a specific functional form. Because of the possibility of postselection, one has to consider different paths given by the outcomes of the generalized measurements, that is, which Kraus operators $K_{j}^{\alpha_{j}}$ have been measured; after $t$ maps (labeled from 1 to $t$ ), these paths are denoted by $\vec{\alpha}_{t}$, and $\alpha_{t+1}$ denotes the possible outcomes of the map labeled by $t+1$. Both the maps and the length of the protocol may vary depending on the outcomes of previous measurements, but for any path $\vec{\alpha}$, the total length $N=N(\vec{\alpha})$ can be restricted to be less than or equal to the dimension of the Hilbert space of the wire. This finiteness of the protocol is proven by showing that one can find a protocol that bounds the support on the wire of each branch $\vec{\alpha}_{N}$, in each step $t$, by a monotonically decreasing sequence of integers $r\left(\vec{\alpha}_{t-1}\right)$. Figure 3 depicts a generic example for a protocol that acts on an initially threedimensional wire and a quantum system.

Proposition 3. Let us denote by $\Lambda_{1}$ a selective CPTP map with outcomes $\alpha_{1}$ represented by Kraus operators $K_{1}^{\alpha_{1}}$. Depending on the obtained outcome $\alpha_{1}$, we define the next selective CPTP map $\Lambda_{2}\left(\alpha_{1}\right)$, with outcomes $\alpha_{2}$, represented by Kraus operators $K_{2}^{\alpha_{2}}\left(\alpha_{1}\right)$. In the same way, we define $\Lambda_{t}\left(\vec{\alpha}_{t-1}\right)$, which depends on the outcomes of all previous maps through $\vec{\alpha}_{t-1}=\left(\alpha_{1}, \ldots \alpha_{t-1}\right)$. For notational convenience, we define $\vec{\alpha}_{0}=\vec{\alpha}_{-1}=0$.

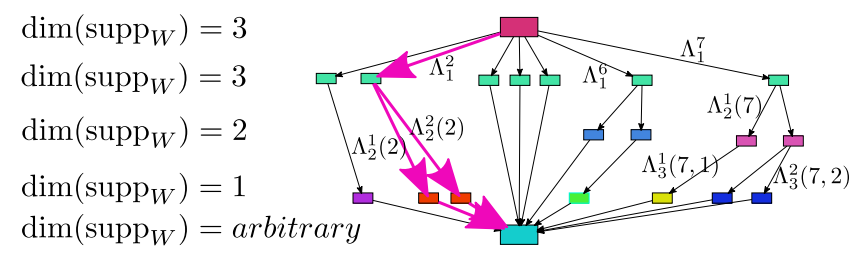

FIG. 3. The figure shows a diagram for a possible protocol defining a LOP map as described in Proposition 3. In this example, the input state is three-dimensional on the wire. All the arrows starting from the same node represent a CPTP map. Each arrow represents a submap with a given outcome (labeled from 1 on the left to $n$ on the right). The dimension of the maximal support of the input or output states on the wire is stated on the left of the diagram. The branches can be grouped into four different types, where the types differ by how the dimension of the maximal support of the state on the wire [given without loss of generality by $r\left(\vec{\alpha}_{t-1}\right)$, after applying $t$ maps with the outcomes $\left.\vec{\alpha}_{t}\right]$ changes during the protocol. Any possible branch type is depicted at least once. In this picture, the respective groups of outcomes for the four branch types are (we only name the outcome vectors up to the point that the group is defined) $\{(1),(2)\} ;\{(3),(4),(5)\}$; $\{(6,1)\}$; and $\{(6,2),(7)\}$. See the Appendix F for more details on the branch types. The highlighted branch is given by $\Lambda_{2}(2) \circ \Lambda_{1}^{2}$, that is, the CPTP map $\Lambda_{2}(2)=\Lambda_{2}^{1}(2)+\Lambda_{2}^{2}(2)$ (composed by two submaps), after getting the outcome 2 in the first map. The length of the protocol $N$ is 2 for these outcomes, while it is 3 for, e.g., the outcomes $(7,2)$. In principle, the dimension of the support of the outcome state on the wire after applying a LOP map is arbitrary (though, for the depicted protocol, it is at most $3 \times 3+1 \times 2+7 \times 1=18$ ). 
Let $\Lambda$ be a CPTP map acting on $W \otimes Q$. Then $\Lambda$ is a LOP operation exactly if it can be written as

$$
\Lambda=\Lambda_{N}\left(\vec{\alpha}_{N-1}\right) \circ \ldots \circ \Lambda_{2}\left(\alpha_{1}\right) \circ \Lambda_{1},
$$

with

$$
K_{t}^{\alpha_{t}}\left(\vec{\alpha}_{t-1}\right)=\sum_{i=1}^{r\left(\vec{\alpha}_{t-2}\right)}\left|\sigma_{\vec{\alpha}_{t}} \circ \min \left[r\left(\vec{\alpha}_{t-1}\right), i\right]\right\rangle\left\langle\left. i\right|_{W} \otimes E_{t}^{\alpha_{t}}\left(\vec{\alpha}_{t-1}, i\right),\right.
$$

where $\sigma_{\vec{\alpha}_{t}}$ is an injective map to the positive labels of a new incoherent basis (see footnote [62]); $E_{t}^{\alpha_{t}}\left(\vec{\alpha}_{t-1}, i\right)$ is an arbitrary operator acting on $Q$, potentially also depending on previous outcomes and controlled by the populations of the wire; and $1 \leq r\left(\vec{\alpha}_{t}\right)<r\left(\vec{\alpha}_{t-1}\right)$ for $t \geq 0$, with $r(0)=\operatorname{dim}(W)$. Therefore, the length of the protocol $N$, possibly depending on the outcomes $\alpha_{t}$ that one obtains, is bounded by $N \leq \operatorname{dim}(W)$.

The above proposition is useful to connect other theories that have been discussed in the literature with the one presented here. From Proposition 3, it follows that destructive measurements on any subsystem $W_{s}$ of $W$ are free (destructive measurements being a set of Kraus operators mapping to a one-dimensional Hilbert space, which consists of only one, trivially incoherent, state). This can also be more directly seen, as one can forward the subsystem $W_{s}$ from the wire to the quantum side and perform the measurement there. Note that one can understand any positive operator valued measure (POVM) as a destructive measurement, since one is not interested in the outcome [65]. This can be stated as "any POVM can be implemented inside LOP." Also, note that any strictly incoherent operation (SIO) can be performed on $W$. Strictly incoherent operations have Kraus operators that commute with dephasing, forcing them to have the form $F^{\alpha}=\sum_{i} c_{\alpha}(i)\left|\sigma_{\alpha}(i)\right\rangle\langle i|$, for permutations $\sigma_{\alpha}$ and complex $c_{\alpha}(i)[24,39]$. Even more restrictive, physical incoherent operations (PIO) are strictly incoherent operations, where the permutations are fixed for all the Kraus operators $\left(\sigma_{\alpha}=\sigma\right)$ [40]. Both are obviously special cases of the form given in Proposition 3. Indeed,

Proposition 4 (SIO, PIO, and LOP). Let $\Lambda$ be a CPTP map acting on $W \otimes Q . \Lambda$ is a LOP operation exactly if it can be written as a sequence of maps $\Lambda=\Lambda_{M}\left(\vec{\alpha}_{M-1}\right) \circ \ldots \circ \Lambda_{1}$, for some finite $M$, where each $\Lambda_{i}$ is

(a) a physical incoherent operation on $W$ or

(b) a destructive measurement in one fully coherent basis of a subsystem of $W$ or

(c) a controlled unitary $\left[\mathbf{U}_{\text {control }}=\sum_{m}|m\rangle\left\langle\left. m\right|_{\text {control }} \otimes\right.\right.$ $\mathbf{U}_{\text {target }}(m)$ ] with control $W$ and target $Q$ or

(d) a generalized measurement of $Q$, encoding the result on $W\left(\rho \mapsto \sum_{\alpha}|\alpha\rangle\left\langle\left.\alpha\right|_{W} \otimes K^{\alpha} \rho K^{\alpha \dagger}\right)\right.$.

One can equivalently replace the item (a) by "a strictly incoherent operation on $W^{\prime \prime}$.
Noting that items (a), (c), and (d) together form an effective theory of the strictly incoherent operations by restricting the theory to the effect on the wire [39], we find that the present approach (apart from giving a completely different motivation) only differs by allowing measurements. This difference, however, is crucial; that strictly incoherent operations commute with dephasing means that, apart from being unable to create coherences, they affect populations only depending on populations, making them incoherent in a very strict sense. So strict, in fact, that coherences do not have any effect that can be measured by free operations. This means that coherences are useless for any observable task in that resource theory and, hence, there are no resource states in the theory. In contrast, one would expect that a meaningful resource helps to overcome the restriction imposed by the resource theory. In this case, the questions of how much resources one needs for a given task make sense. Ideally, but maybe not always necessarily, one would find that having enough nonfree states at hand removes completely any restrictions of the theory. Exactly this we find in the present approach: If supplemented by enough coherent ancillary states, LOP can be used to achieve any desired quantum operation.

Theorem 5 (Resource states). Let $\Lambda$ be a CPTP map acting on $W_{1} \otimes Q$, with $W_{1}$ having dimension $d$. Let $|\psi\rangle=$ $\sum_{i=1}^{d}(1 / \sqrt{d})|i\rangle$ be a maximally coherent state on $W_{2}$. Then, there is an operation $\Lambda^{\prime} \in \operatorname{LOP}\left(W_{2} \otimes W_{1} \stackrel{\aleph}{\leftarrow}\right)$, with $\operatorname{Tr}_{W_{2}}\left[\Lambda^{\prime}\left[|\psi\rangle\left\langle\left.\psi\right|_{W_{2}} \otimes \rho_{W_{1}, Q}\right]\right]=\Lambda\left[\rho_{W_{1}, Q}\right]\right.$.

We conclude that coherence is a meaningful resource in LOP. Furthermore, from Lemma 2, it follows that the resource of coherence $\sum_{i=1}^{d}(1 / \sqrt{d})|i\rangle_{W}$ can be reversibly converted into entanglement between the wire and the system $Q, \sum_{i=1}^{d}(1 / \sqrt{d})|i i\rangle_{W Q}$, which, hence, is an equivalent resource of the theory (also see Refs. [26,43]). Note that the wire and the quantum systems are not spatially separated. Therefore, the interpretation of entanglement as nonclassical correlation over a distance-as it arises naturally in the LOCC setting -is less fruitful. One may then wonder what entanglement between the wire and the system $Q$ means and which implications it may have. As a reminder, let us first look more closely at the interpretation of entanglement in the case of LOCC. If the system is in a maximally entangled state, quantum mechanics predicts strong correlations between different sets of possible local measurement outcomes. These correlations can relate in a way that is not possible classically (in a local hidden-variable theory), due to the distance of the parties [6]. In LOCC, one assumes to be able to keep, measure, and manipulate the state locally and coordinate the manipulations by classical communication (these are the free operations). Therefore, entanglement between two distant parties gives an upper bound to the ability of using and manipulating nonclassical correlations. On the other hand, for entanglement between a quantum system and a wire in LOP, we are more restricted in the 
manipulation. Instead, the wire is thought to be an extended system and is connected to the quantum system over which one has full quantum control. So that, in this case, the entanglement gives a bound to the ability to spread and measure the strong correlations and, by doing so, making them provably nonclassical. Additionally, one can use any resource state to coherently control the wire [43] and, for instance, steer its state [31].

The above properties closely resemble those found for incoherent-quantum operations (IQO) [42] and, as stated above, the free operations of this theory contain LOP. But how strict is this inclusion? In the following proposition, we show that, in general, incoherent-quantum operations cannot be performed by LOP operations. However,

Proposition 6 (LOP and IQO). Be $\Lambda$ an incoherentquantum operation on $W_{1} \otimes Q$, which is exactly the case if it is CPTP and has a Kraus decomposition with Kraus operators of the form $K^{\alpha}=\sum_{i}\left|f_{\alpha}(i)\right\rangle\left\langle\left. i\right|_{W_{1}} \otimes E^{\alpha}(i)\right.$, for some functions $f_{\alpha}$ acting on the labels of the incoherent basis and some operators $E^{\alpha}(i)$ acting on $Q$. Let $d:=\operatorname{dim}\left(W_{1}\right)$. If $d=2, \Lambda \in$ LOP. For $d \geq 3$, LOP $\neq$ IQO, but there is a stochastic implementation of the map in LOP with a success rate of at least $1 / d$; i.e., there is an operation $\Lambda^{\prime} \in \operatorname{LOP}\left(W_{2} \otimes W_{1} \rightsquigarrow Q\right)$ with $\Lambda^{\prime}\left[|0\rangle\left\langle\left. 0\right|_{W_{2}} \otimes \rho\right]=|0\rangle\left\langle\left. 0\right|_{W_{2}} \otimes \Lambda_{0}^{\prime}[\rho]+\mid 1\right\rangle\left\langle\left. 1\right|_{W_{2}} \otimes \Lambda_{1}^{\prime}[\rho]\right.\right.$ with $\Lambda_{1}{ }^{\prime}[\rho]=\Lambda[\rho] / d \quad \forall \rho$.

This means that, even though the theories LOP and IQO differ, they are very similar. Equally close are the theories they induce on the wire (by tracing out $Q$ at the end). We conclude that the effective theory we obtain by restricting LOP to the wire- even though being operationally motivated-is, in many aspects, similar to the abstractly motivated theory of coherence introduced in Ref. [23], where the free operations are any that can be decomposed into Kraus operators that cannot create coherence. Specifically, the free states are the same, the resource states are the same, and the same amount of resources is needed to remove all restrictions of the theories; in both theories, any destructive measurements can be performed; for qubits, the theories are equivalent; and for higher dimensions, the theories are stochastically equivalent.

Distillation Furthermore, for a given $\epsilon$, having enough copies of any state that is not incoherent quantum, LOP allows us to prepare a maximally coherent state with fidelity $f>1-\epsilon$ and probability $p>1-\epsilon$ (see Appendix E). Therefore, since maximally coherent states can be used as a resource to implement any quantum operation (Theorem 5), all not incoherent-quantum states are resources for nonclassicality of the wire in the present setting. This is true independently of how one defines nonclassicality, as long as it does not include full quantum theory.

More specifically, one can make a stochastic model for the evolution of the wire for any free initial state and under any free operation. This can be done in a way that the stochastic model correctly describes the change of the reduced state of the wire, as long as at the beginning the full state is incoherent quantum. Crucially, this model does not need to depend on the populations (in the incoherent basis) of the wire. However, it may depend on the initial state of $Q$, conditioned on the populations of the wire. This is fine, since $Q$ is not part of the description of the system we are modeling classically (which is the wire $W$ ), but interacts with it. So, it is in agreement with a classical picture that the state of $Q$ may change the transition probabilities. That is, the state of $Q$, together with its evolution and possible measurements, defines external parameters of the model. Note that it is not an issue, that it may not be possible to describe the evolution of $Q$ by a classical model. We do not need to describe the full evolution, we only need the conditional probabilities $p(\omega \mid i)$ that, given that the state of the wire at the beginning was the pure incoherent state $|i\rangle$, a measurement (scheduled by the predefined protocol) of $Q$ will yield the outcome $\omega$ (which is the only part which-by encoding - affects the wire). Since the conditional initial states of $Q$ (conditioned on the populations $|i\rangle$ of the wire) are fixed, these probabilities are fixed as well, and there is no need to model the full evolution of $Q$. Explicitly, one just needs to replace the elemental operations, that is, permutations, tracing out and encoding a classical outcome of a measurement by their obvious noncontextual (and local) classical counterparts. For the measurements, this means encoding the classical probabilities as defined by the Born rule and the current state of the external quantum system to measure, conditioned on the previous populations of the wire: its previous classical states. If the initial state was not incoherent quantum, by forwarding the state of the wire to the quantum side and measuring it, coherences of the wire can affect the measurement probabilities. Then, the simple model given above is no longer sufficient to describe the evolution of the populations of the wire throughout the full protocol. Indeed, the argument above shows that this difference is maximal, in that having enough copies of any state that is not incoherent quantum will allow us to do any operation on the full system, including those that cannot
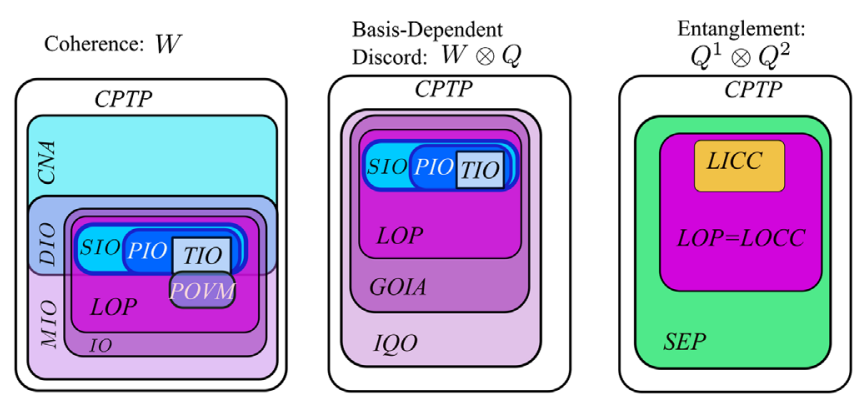

FIG. 4. Venn diagram for the inclusion relations of LOP with selected coherence theories (left), theories of basis-dependent discord (middle), and entanglement theories (right), within general CPTP operations (see Ref. [25] for additional classes). See Appendix B for a description of the different classes and their relations. 
be modeled by a noncontextual (or local; see the next section about entanglement) classical model.

\section{Quantum discord}

Before continuing and applying the tools we have just developed to multipartite entanglement, let us stop a moment and draw the connection to quantum discord [18-21]. In a sense to be made precise now, LOP can be seen as a theory of quantum discord. As the free states of LOP are the incoherent-quantum states defined above, the theory can be seen as quantifying how much a state differs from an incoherentquantum state, that is, by definition, how much basisdependent quantum discord it has (called measurement dependent in Ref. [20]). Take any measure of basis-dependent discord that can, at the same time, be normalized such that it yields 1 for a singlet state. Assume also that the measure is upper bounded by the entropy of the local state of the wire. Then, by minimizing this quantity over all possible bases, we obtain a measure of discord satisfying the properties stated in Sec. III. A. 1. of the review [20] (this was noted before in Refs. [42,43]). While starting from coherence theory, it seems strange to do this optimization, since one starts by choosing a natural basis as incoherent, and only then does the notion of coherence make sense; the present approach is much nearer to one original setting in which quantum discord was introduced [18]. Indeed, the theory of coherence of the wire in the present approach is an effective theory, and the incoherent basis is arbitrary; it just depends on what one assumes to be the default basis in which to encode the information. This allows us to interpret quantum discord as a natural lower bound that defines the nonclassical resource one has to assume, at least, independently of the basis chosen. However, this is not the same as to say that quantum discord is a resource of nonclassicality. What happens is that, if one has two quantum states that have zero discord, there still might be no basis for which both have zero basis-dependent discord simultaneously and it is, thus, not surprising that their mixture might not be discord free (also see the discussion in Ref. [18] and Fig. 2 in Ref. [43]).

This means that, in the present framework, we reproduce the interpretation of quantum discord as how nonclassical one has to assume the state of the wire $W$ at least. This bound is tight when $W$ is seen as a part of the full system $W \otimes Q$ (similarly as was the motivation for the introduction of quantum discord in [18]) and if one considers only one state of $W$ at a given time (though one can consider different states at different times, since, as noted above, one can change the basis in time). But having more than one possible state (for instance if one does not consider the evolution of one given state, but a protocol defined for any input state, such as in quantum cryptography), it is necessary to fix one basis (possibly a different one at different times) for all the states one considers, to have a meaningful quantity.

In summary, discord is not a resource; it is an indicator of nonclassicality: If a state has nonzero discord, this means that, for whatever basis of the wire one chooses as incoherent, the state is a resource for nonclassicality with the present framework. It is in this sense that the present approach defines a resource theory of quantum discord, even though discord is not (and should not be) a monotone and, thus, a measure of the resource theory.

\section{COHERENCE COST OF ENTANGLEMENT}

The aim of this section is to better understand multipartite entanglement by looking at the coherence needed to generate it. This is a natural approach, as entanglement is always a result of coherent interactions that happened in the past. In the case of bipartite entanglement, the theory in our setting is the one depicted in Fig. 1, which we denote by $\operatorname{LOP}\left(Q^{1 \stackrel{\leftrightarrow}{\rightarrow}} W \stackrel{\rightsquigarrow}{\leftarrow} Q^{2}\right)$, and consists of two quantum systems $Q^{1}$ and $Q^{2}$ connected by a wire $W$. The elemental free operations are the free operations of $\operatorname{LOP}\left(W \rightsquigarrow Q^{1}\right)$, together with the ones in $\operatorname{LOP}\left(W \rightsquigarrow Q^{2}\right)$, meaning that any operation is given by the composition of these operations, possibly with postselection. In general, we

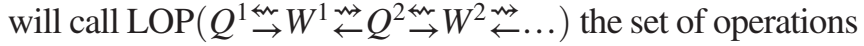
consisting of concatenating operations in the corresponding

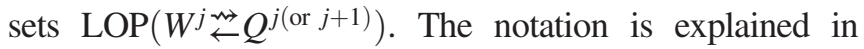
Fig. 5.

The minimal amount of coherence needed to create pure bipartite entanglement directly follows from Lemma 2 (also see Ref. [26]). One can produce a pure state on $Q^{1} \otimes Q^{2}$, which, in its Schmidt decomposition [65], is given by $|\psi\rangle_{Q^{1}, Q^{2}}=\sum_{i} c_{i}|i i\rangle_{Q^{1}, Q^{2}}$ from $\sum_{i} c_{i}|i\rangle_{W}$ on $W$ by applying Lemma 2 to get $|\psi\rangle_{Q^{1}, W}$ and then using classical to quantum forwarding on $W \otimes Q^{2}$ to get the wanted state. On the other hand, one also sees that the production is optimal, since one needs to have at least that amount of entanglement on the bipartite cut $Q^{1} \mid W \otimes Q^{2}$ [remember that entanglement and coherence are equivalent resources for $\left.\operatorname{LOP}\left(Q^{1 \stackrel{\text { m}}{\rightarrow}} W\right)\right]$.

As shown below, the connection between coherence and entanglement in the bipartite case is even stronger; a maximally correlated state can be used as a resource for a LOCC transformation exactly if the equivalent coherent state can be used as a resource to do the transformation under

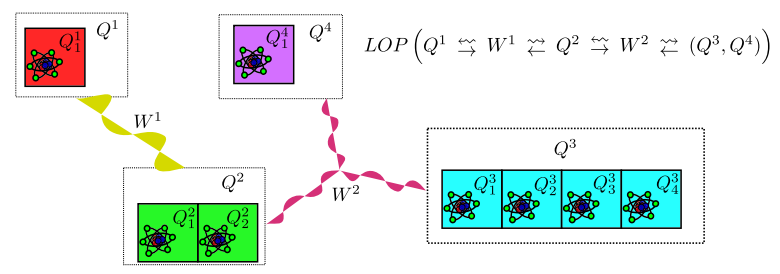

FIG. 5. The figure shows a possible wiring for four parties. The first wire connects the systems $Q^{1}$ and $Q^{2}$, and the second connects $Q^{2}, Q^{3}$, and $Q^{4}$, resulting in the theory LOP $\left[Q^{1 \stackrel{\leftrightarrow}{\rightarrow}} W^{1} \stackrel{\rightsquigarrow}{\leftarrow} Q^{2 \stackrel{\leftrightarrow}{\rightarrow}} W^{2} \stackrel{\aleph}{\leftarrow}\left(Q^{3}, Q^{4}\right)\right]$. Each party (and also the wires) might consist of more than one quantum system, and changing the number of subsystems of each party is a free operation in LOP. 
LOP. This puts the connection between coherence and entanglement that has recently attracted a lot of attention on the level of resources and operations instead of measures [26-42]. It will be useful to introduce the notation $W^{j}=$ $W_{1}^{j} \otimes W_{2}^{j} \ldots$ and $Q^{j}=Q_{1}^{j} \otimes Q_{2}^{j} \ldots$, with the convention that the upper index labels the local systems and the lower their respective subsystems, whose number may vary. The full systems are referred to by $Q=\otimes_{j} Q^{j}, W=\otimes_{j} W^{j}$.

Theorem 7. Let $\eta_{\text {LOCC }}=\sum_{i j} r_{i, j}|i\rangle\left\langle\left. j\right|_{Q_{1}^{1}} \otimes \mid i\right\rangle\left\langle\left. j\right|_{Q_{1}^{2}}\right.$ be a maximally correlated state (in arbitrary orthonormal local bases of $Q_{1}^{1} \otimes Q_{1}^{2}$ ) and $\eta_{\mathrm{LOP}}=\sum_{i j} r_{i, j}|i\rangle\left\langle\left. j\right|_{W}\right.$ be a corresponding state in the incoherent basis $\mathcal{Z}$ of $W$. If $\Lambda$ is a CPTP map on $Q^{1} \otimes Q^{2}$, then the following statements are equivalent:

$$
\begin{aligned}
\text { 1. } & \exists \Lambda_{\text {LOCC }} \in \operatorname{LOCC}\left(Q^{1}, Q^{2}\right): \\
& \Lambda_{\text {LOCC }}\left[\eta_{\text {LOCC }} \otimes \rho_{Q_{2}^{1}, Q_{2}^{2}}\right]=\Lambda\left[\rho_{Q_{2}^{1}, Q_{2}^{2}}\right] \quad \forall \rho_{Q_{2}^{1}, Q_{2}^{2}}
\end{aligned}
$$$$
\text { 2. } \exists \Lambda_{\mathrm{LOP}} \in \operatorname{LOP}\left(Q^{1 \stackrel{\leftrightarrow}{\rightarrow}} W \stackrel{\rightsquigarrow \leftrightarrow}{\leftarrow} Q^{2}\right):
$$$$
\Lambda_{\mathrm{LOP}}\left[\eta_{\mathrm{LOP}} \otimes \rho_{Q_{2}^{1}, Q_{2}^{2}}\right]=\Lambda\left[\rho_{Q_{2}^{1}, Q_{2}^{2}}\right] \quad \forall \rho_{Q_{2}^{1}, Q_{2}^{2}} .
$$

We now use the common definition that $\rho \stackrel{\mathcal{O}}{\rightarrow} \sigma$ means that there is a map $\Lambda \in \mathcal{O}$, with $\Lambda[\rho]=\sigma$ (for some space of superoperators $\mathcal{O}$ ). The following corollary then follows directly by taking $\Lambda$ in the theorem to be the preparation of the state $\sigma_{Q^{1}, Q^{2}}$ :

Corollary 8. Let $\eta_{\mathrm{LOCC}}=\sum_{i j} r_{i, j}|i\rangle\left\langle\left. j\right|_{Q_{1}^{1}} \otimes \mid i\right\rangle\left\langle\left. j\right|_{Q_{1}^{2}}\right.$ be a maximally correlated state (in arbitrary orthonormal local bases) and $\eta_{\text {LOCC }}=\sum_{i j} r_{i, j}|i\rangle\left\langle\left. j\right|_{W}\right.$ be a corresponding state in the incoherent basis $\mathcal{Z}$ of $W$. Then,

$$
\begin{aligned}
& \eta_{\text {LOCC }} \otimes \rho_{Q_{2}^{1}, Q_{2}^{2}} \stackrel{\operatorname{LOCC}\left(Q^{1}, Q^{2}\right)}{\longrightarrow} \sigma_{Q^{1}, Q^{2}}
\end{aligned}
$$

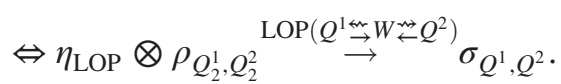

Having shown this very strong connection between coherence and bipartite entanglement, we move to the multipartite LOCC case. While, in the bipartite case, it is quite clear how one needs to explicitly implement the wires (as there is one clearly simplest way to connect two parties), in the multipartite case, the situation is more complex. To be able to do any operation by using enough coherence, each party needs to be connected to all others (possibly indirectly over third parties). On the other hand, one also does not need to have two parties connected in two different ways, and avoiding double connections simplifies the theories. We use the shorthand notation $\operatorname{LOP}\left[W^{1} \stackrel{\rightsquigarrow}{\leftarrow}\left(Q^{1}, Q^{2}, \ldots\right)\right]$ for the case of one wire connecting different quantum systems [i.e., concatenating operations in the sets $\left.\operatorname{LOP}\left(W^{1} \stackrel{\aleph}{\leftarrow} Q^{i}\right)\right]$. For the example of three parties $Q=A \otimes B \otimes C$, we are left with the $3+1$ theories of the two types depicted in Fig. 6, namely, the three ways $\left(A, B\right.$, or $C$ ) of choosing $Q^{2}$ in

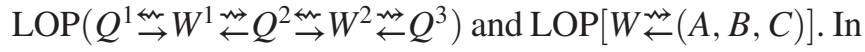
general, we get that, following the rules explained above, the (a)

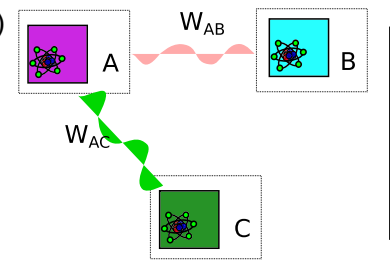

(b)

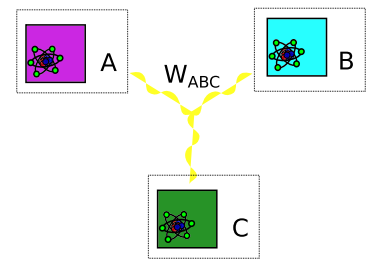

FIG. 6. Two wiring schemes that are equivalent in the LOCC paradigm, but inequivalent according to LOP. For LOCC, i.e., for incoherent states of the wiring, every operation can be performed by acting over the extremes of each wire, without a direct interaction among wires. On the other hand, if wires are quantum, direct interaction among wires enlarges the set of possible inequivalent operations. As a result, the initial coherence necessary to prepare a multipartite entangled state depends on the wiring topology.

possible generalization of $N$-partite LOCC is exactly given by the theories

$$
\begin{aligned}
\operatorname{LOP} & {\left[\left(Q^{\sigma(1)} \ldots Q^{\sigma\left(f_{1}\right)}\right)_{\stackrel{\leftrightarrow}{\rightarrow}}^{\rightarrow} W^{1} \stackrel{\rightsquigarrow}{\longleftarrow}\left(Q^{\sigma\left(f_{1}+1\right)} \ldots Q^{\sigma\left(f_{2}\right)}\right)\right.} \\
& \left.\stackrel{\leftrightarrow n}{\rightarrow} W^{L} \stackrel{\rightsquigarrow}{\longleftrightarrow}\left(Q^{\sigma\left(f_{L}+1\right)} \ldots Q^{\sigma(N)}\right)\right],
\end{aligned}
$$

where $\sigma$ is a permutation of the indexes denoting the local quantum systems. Of course, one could also look at the union of all these theories, which has the advantage of providing a completely unified view, while having the disadvantage of being excessively complicated. What all these theories have in common is that they are generalizations of multipartite LOCC on the quantum side, that reduce to it if one starts with an incoherent state on $W$, which is made precise in the following remark.

Remark 9. $\forall \rho_{W}=\sum_{i} p_{i}|i\rangle\left\langle\left. i\right|_{W}\right.$,

$$
\begin{aligned}
& \text { 1. } \operatorname{Tr}_{W}\left[\Lambda \circ\left(\rho_{W} \otimes \mathbb{1}_{Q}\right)\right] \in \operatorname{LOCC}\left(Q^{1}, \ldots, Q^{n}\right)
\end{aligned}
$$

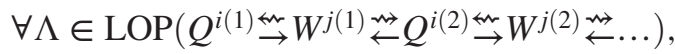

$$
\begin{aligned}
& \text { 2. } \Lambda \otimes \mathbb{1}_{W} \in \operatorname{LOP}\left(Q^{i(1) \stackrel{\leftrightarrow}{\rightarrow}} W^{j(1)} \stackrel{\rightsquigarrow}{\longleftarrow} Q^{i(2) \stackrel{\leftrightarrow}{\rightarrow}} W^{j(2)} \rightleftarrows \ldots\right) \\
& \forall \Lambda \in \operatorname{LOCC}\left(Q^{1}, \ldots, Q^{n}\right) \text {, }
\end{aligned}
$$

with $i$ and $j$ denoting permutations on the index sets.

In the bipartite case, this means that, if the initial state of the wire is incoherent and the wire is in a product state with both parties, tracing out the wire at the end reduces the theory to LOCC. The reason is that the state of the wire can be copied and stored in a local register on both sides. Every operation above can then just be obtained by local operations and broadcasting of classical information, by updating the two local registers (just do the same permutations and phases on both registers, copy the information of measurements to both registers, and trace out the corresponding part of a register on Bob's side if Alice's has been used in a quantum operation). We make this seemingly obvious statement precise, because intuition sometimes might be misleading; for instance, it is possible 
to produce entanglement by sending a quantum particle back and forth that is never entangled with either of the two parties [54]. While, in entanglement theory, this is surprising, since one can entangle two parties without using entanglement to do so, with the present approach, the same statement is very intuitive, as entanglement is simply not the only resource present; coherence and basis-dependent discord are resources as well. In the present approach, without having resources, one cannot entangle two parties, while any amount of basis-dependent discord allows us to do that (that is because, as noted above, one can distillate coherence from any resource and the statement then just follows from Theorem 7). The above remark makes it easy to prove that the coherence needed to create entanglement gives a bound on the possible entanglement conversions, namely,

Theorem 10. Let $\rho, \sigma$ be states on $W$. If $\exists \tau_{W}$, a state on $W$, s.t. $\tau_{W} \stackrel{\operatorname{LOP}\left(Q^{i(1)} \stackrel{\text { m }}{\rightarrow} W^{j(1)} \ldots\right)}{\rightarrow} \rho$, but $\tau_{W} \stackrel{\operatorname{LOP}\left(Q^{i(1)} \stackrel{\text { m }}{\rightarrow} W^{j(1)} \ldots\right)}{\rightarrow}$, then it follows that $\rho \stackrel{\operatorname{LOCC}\left(Q^{1}, \ldots, Q^{n}\right)}{\leftrightarrow} \sigma$.

Note that, for any of these theories (independently of the wiring), the free states are given by

$$
\begin{aligned}
C Q_{\mathcal{Z}^{\otimes}}^{(n)} & =\left\{\rho\left|\rho=\sum_{m} p_{m}\right| m\right\rangle\left\langle m\left|\otimes \rho_{m},\right| m\right\rangle \\
& \left.\in \mathcal{Z}^{\otimes}, \rho_{m} \in \operatorname{SEP}(n)\right\},
\end{aligned}
$$

with $\mathcal{Z}^{\otimes}$ the product basis of the incoherent basis of each wire, and $\operatorname{SEP}(n)$ the set of $n$-partite separable states,

$$
\operatorname{SEP}(n)=\left\{\rho \mid \rho=\sum_{k} p_{k} \rho_{1}^{(k)} \otimes \ldots \otimes \rho_{n}^{(k)}\right\} .
$$

This implies that, for any of these theories,

$$
R_{\mathcal{Z}^{\otimes}, n}(\rho)=\min _{\sigma \in C Q_{\mathcal{Z}^{\otimes}}^{(n)}} S(\rho \| \sigma),
$$

with $S(\rho \| \sigma):=\operatorname{Tr}(\rho[\log (\rho)-\log (\sigma)])$ the relative entropy, defines an additive geometric monotone (see, e.g., Ref. [43]). In general, the evaluation of this quantity is an nondeterministic polynomial time (NP)-complete problem [66]. However, because of the monotonicity of the relative entropy, we notice that

$$
R_{\mathcal{Z}^{\otimes}, n}(\rho) \geq \max \left[R_{\mathcal{Z}^{\otimes}}\left(\operatorname{Tr}_{Q} \rho\right), R_{n}^{\mathcal{E}}\left(\operatorname{Tr}_{W} \rho\right)\right]
$$

with $R_{\mathcal{Z}^{\otimes}}\left(\operatorname{Tr}_{Q} \rho\right)$ the relative entropy of coherence on the wires, and $R_{n}^{\mathcal{E}}\left(\operatorname{Tr}_{W} \rho\right)$ the relative entropy of entanglement $[48,67,68]$. Because of the additivity of the relative entropy, if either $R_{\mathcal{Z}^{\otimes}}\left(\operatorname{Tr}_{Q} \rho\right)=0$ or $R_{n}^{\mathcal{E}}\left(\operatorname{Tr}_{W} \rho\right)=0$, Eq. (4) turns into an equality, providing a lower bound to the amount of initial coherence required to prepare an entangled state among the parties of $Q[26,42]$.

As an example of how this perspective can be applied in the multipartite case, let us revisit the case of the $|\mathcal{W}\rangle$ and of the $|G H Z\rangle$ state [55] (here, only the results are discussed; the protocols for the conversions can be found in Appendix D).

The relative entropy of entanglement of $\left|G H Z_{n}\right\rangle=$ $\left[\left(|0\rangle^{\otimes n}-|1\rangle^{\otimes n}\right) / \sqrt{2}\right]$ is 1 [67] and, indeed, one can prepare the $\left|G H Z_{n}\right\rangle$ state by $\operatorname{LOP}\left[W_{\leftarrow}^{\curvearrowleft}\left(Q^{1}, Q^{2}, \ldots, Q^{n}\right)\right]$ from $(|0\rangle+|1\rangle) / \sqrt{2}$. For $\left|\mathcal{W}_{n}\right\rangle=\left[\left(\sum_{k=0}^{n-1}|0\rangle^{\otimes k}|1\rangle|0\rangle^{\otimes n-k-1}\right) /\right.$ $\sqrt{n}]$, the relative entropy of entanglement is $(n-1) \log _{2}[n /$ $(n-1)]>1 \forall n>2$ [68]. It is then a simple corollary of Theorem 10 that $\left|G H Z_{n}\right\rangle^{\operatorname{LOCC}\left(Q^{2}, \ldots, Q^{n}\right)}\left|\mathcal{W}_{n}\right\rangle$.

The second thing to note is that, on any bipartition, the $\left|G H Z_{n}\right\rangle$ state is LOCC equivalent to $\left|G H Z_{2}\right\rangle$, while the $\left|\mathcal{W}_{3}\right\rangle$ state on any bipartition is LOCC equivalent to $1 / \sqrt{3}(|00\rangle+\sqrt{2}|11\rangle)$. Indeed, one can, in all three pos-

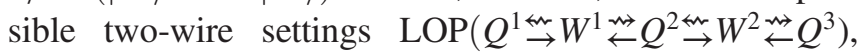
prepare $\left|\mathcal{W}_{3}\right\rangle$ from $1 / \sqrt{6}(|0\rangle+\sqrt{2}|1\rangle)_{W^{1}} \otimes(|0\rangle+|1\rangle)_{W^{2}}$, while the bipartite entanglement one can produce on the bipartition $Q^{1},\left(Q^{2} \otimes Q^{3}\right)$ is not enough to prepare $\left|G H Z_{3}\right\rangle$ : As on any bipartition, one needs to prepare a fully entangled qubit, and this is equivalent to a maximally coherent qubit; the state with minimal coherence to prepare $\left|G H Z_{3}\right\rangle$ is given by $1 / 2(|0\rangle+|1\rangle)_{W^{1}} \otimes(|0\rangle+|1\rangle)_{W^{2}}$, which is strictly more coherent on $W_{1}$ than $1 / \sqrt{6}(|0\rangle+$ $\sqrt{2}|1\rangle)_{W^{1}} \otimes(|0\rangle+|1\rangle)_{W^{2}}$. Again, as a corollary of

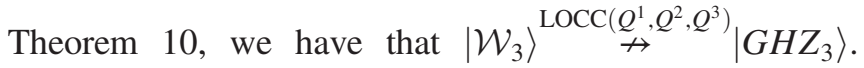
This is a strong indication that the resources in the different types of wirings correspond to different types of entanglement.

\section{CONCLUSION}

Recently, there has been considerable interest in the connections among coherence, discord, and entanglement [26-42]. But the connection was made on the level of quantifiers and measures. The current paper shows that, by generalizing the fundamental theory of entanglementLOCC, one obtains connections among coherence, discord, and entanglement that are even deeper, namely, on the level of the operations themselves. We call this generalized version of LOCC "local operations and physical wires" (LOP). In this sense, LOP lies at the root of entanglement, and it seems natural to assume that it will be useful to assess the interplay between different resources of quantumness in complex settings, as is necessary, for instance, if one wants to quantify the resources needed for quantum algorithms (see, e.g., Ref. [69]). Moreover, while we exemplified here that LOP can give a clear explanation of the difference between some very basic forms of multipartite entanglement by exemplifying that different forms are optimal in different settings, it remains an interesting open question whether it yields such an explicative power also in more general cases 
and how it connects to known structures in entanglement theory, such as explained in, e.g., Ref. [56-60].

Furthermore, there is an ongoing discussion over what is the "right" theory of (speakable) coherence [36, 39-41,70,71]. While we do not claim to close this discussion (nor actually that it can be conclusively closed, as it always will depend on the setting one is interested in), we note that the effective resource theory of coherence emerging from LOP is, to our knowledge, the first that is built on operationally meaningful elemental operations while still having coherence as a resource in the sense of the name, meaning that enough ancillary coherence can completely lift the restrictions imposed by the resource theory.

In our view, the main contribution of this paper consists in providing a clear picture of the connection between different instances of nonclassicality. While this is principally of interest on a theoretical level, it may also provide insights that are relevant for the description of experimental quantum technologies. This becomes evident, for example, in considering the communication of classical information via a physical channel. While this transfer is typically considered on an abstract mathematical level, treated completely independent of the physical setup, a communication channel is not an abstract notion, but a physical quantum system-a wire. Such a wire, ultimately, is quantum mechanical. The extent to which these quantum properties survive dictates the wire's power as a communication channel and, importantly, its deviation from the idealized mathematical description of classical communication. To this end, having a clean way to treat resources in the wire certainly helps to assess what can and cannot be achieved with such a wire and if the resources are used in an optimal way (or to meaningfully quantify the amount of lost resources in each step of the protocol). It is also worth noting that many proofs in the appendixes are given by explicit protocols, which are built by concatenating elemental operations of the theory and might be used as a starting point to more realistic implementations. As a further application, it is straightforward to adapt LOP to quantify nonclassicality in open quantum systems if the environment can be probed (see the discussion at the end of Sec. III B). Finally, in infinite-dimensional systems, it is often taken as natural to call mixtures of nonorthogonal pure states classical, e.g., mixtures of coherent states of light, since they are usually a lot easier to produce and manipulate (just turn on lasers and use passive linear optics). For this reason, Gaussian channels have been used for secret key sharing (see, e.g., Ref. [72]). While the picture changes (e.g., changing phases should not be free operations; see, e.g., Ref. [45]), we believe that the present approach is a good starting point for developing a reasonable theory of nonclassicality of light.

\section{ACKNOWLEDGMENTS}

We gratefully acknowledge discussions with Andrea Smirne, Ish Dhand, Raúl Rossignoli and Adriana De Mendoza. The work of D.E., T. T., and M. B.P. is supported by the ERC Synergy Grant ERC-2012-SyG BioQ and the U.S. Army Research Office. J. M. Matera is supported by CONICET and the National University of La Plata.

\section{APPENDIX A: PROOFS ON THE STRUCTURE OF LOP}

We start with the proof of Lemma 2, introducing a bijection between the wire and maximally correlated states on the quantum system with the wire within LOP.

Lemma (2). The operator $B: W \rightarrow W \otimes Q, \quad B=$ $\sum_{i}|i\rangle\left\langle\left. i\right|_{W} \otimes \mid i\right\rangle_{Q}$ defines an injection and defining $\mathcal{B}[\rho]=\left(B \rho B^{\dagger}\right), \mathcal{B} \in$ LOP. Conversely there exists a map $\mathcal{B}^{-1}: W \otimes Q \rightarrow W$, with $\mathcal{B}^{-1} \circ \mathcal{B}=\mathbb{1}_{W}$ and $\mathcal{B}^{-1} \in$ LOP.

Proof.-The operator $B=\sum_{i}|i\rangle\left\langle\left. i\right|_{W} \otimes \mid i\right\rangle_{Q}$ can be implemented by a sequence of maps that will be described in the following in terms of their Kraus operators. To this end, we start with $W=W_{1}$ and $Q=1$ and apply

(1) $|0\rangle_{W_{2}}$

(2) $\sum_{i, j}|i\rangle\left\langle\left. i\right|_{W_{1}} \otimes \mid i+j\right\rangle\left\langle\left. j\right|_{W_{2}}\right.$

(3) $\sum_{i, j}\left\langle\left. j\right|_{W_{2}} \otimes \mid j\right\rangle_{Q}$,

where the first map is a single outcome measurement of $Q$ storing the outcome in an ancilla $W_{2}$ (observed quantum operation), the second is a permutation on $W$, and the third is the forwarding of the system $W_{2}$ to $Q$. Identifying $W=W_{1}$, we get the desired operation.

For the converse, we first apply a measurement in the Fourier basis, followed by a correction of the phase on $W$ :

(1) $F T^{k}=\left\langle\left.\hat{k}\right|_{Q}=\sum_{j=1}^{d}\left[\left(e^{2 \pi i k j / d}\right) / \sqrt{d}\right]\left\langle\left. j\right|_{Q}\right.\right.$

(2) $D(k)=\sum_{i=1}^{d}|i\rangle\left\langle\left. i\right|_{W} e^{-2 \pi i k i / d}\right.$,

resulting in the action $B^{-1 k}=\sum_{i=1}^{d} \sum_{j=1}^{d}\left[\left(e^{2 \pi i k(j-i) / d}\right) /\right.$ $\sqrt{d}]|i\rangle\left\langle\left. i\right|_{W} \otimes\left\langle\left. j\right|_{Q}\right.\right.$. Obviously, the map defined by these Kraus operators is an element of LOP and a left inverse of $\mathcal{B}$, as required.

Note that the basis on the quantum side can be chosen arbitrarily in the above lemma. We continue with the proof of Proposition 1.

Proposition (1). Any operation in LOP can be done with an arbitrarily high probability of success by a combination of permutations, observed quantum operations, and classical to quantum forwarding.

Proof.-Let $W$ have dimension $d$. The only thing to show is that, indeed, one can change the phases on $W$ in the above framework. Let $U=\sum_{j} e^{i \phi(j)}|j\rangle\left\langle\left. j\right|_{W}\right.$ be the wanted phase shift. We start by identifying $Q=Q_{1}$. The protocol is

(1) $B=\sum_{i}|i\rangle\left\langle\left. i\right|_{W} \otimes \mid i\right\rangle_{Q_{2}}$ as in Lem 2

(2) $U_{Q_{2}}=\sum_{j} e^{i \phi(j)}|j\rangle\left\langle\left. j\right|_{Q_{2}}\right.$

(3) $F T^{k}=\left\langle\left.\hat{k}\right|_{Q_{2}}=\sum_{j=1}^{d}\left[\left(e^{2 \pi i k j / d}\right) / \sqrt{d}\right]\left\langle\left. j\right|_{Q_{2}}\right.\right.$, $(1 \leq k \leq d)$ 
(4) If $k=d$ : stop.

Else: redefine $U=U \circ \sum_{j} e^{-2 \pi i k j / d}|j\rangle\langle j|$ and restart with item 1.

The probability of success in each round is given by $1 / d$, independently of the initial state. After $M$ iterations, we therefore have a probability of success given by $\sum_{i=1}^{M} 1 / d(1-$ $1 / d)^{(i-1)}=1-[1-(1 / d)]^{M} \rightarrow 1$ for $M \rightarrow \infty$.

The next two pages are devoted to the proof of the closed form of LOP operations, which simplifies its use for both theoretical and practical purposes, for instance, for the comparison of LOP to other resource theories.

Proposition (3). Let us denote by $\Lambda_{1}$ a selective CPTP map with outcomes $\alpha_{1}$ represented by Kraus operators $K_{1}^{\alpha_{1}}$. Depending on the obtained outcome $\alpha_{1}$, we define the next selective CPTP map $\Lambda_{2}\left(\alpha_{1}\right)$, with outcomes $\alpha_{2}$, represented by Kraus operators $K_{2}^{\alpha_{2}}\left(\alpha_{1}\right)$. In the same way, we define $\Lambda_{t}\left(\vec{\alpha}_{t-1}\right)$, which depends on the outcomes of all previous maps through $\vec{\alpha}_{t-1}=\left(\alpha_{1}, \ldots \alpha_{t-1}\right)$. For notational convenience, we define $\vec{\alpha}_{0}=\vec{\alpha}_{-1}=0$.

Let $\Lambda$ be a CPTP map acting on $W \otimes Q$. Then $\Lambda$ is a LOP operation exactly if it can be written as

$$
\Lambda=\Lambda_{N}\left(\vec{\alpha}_{N-1}\right) \circ \ldots \circ \Lambda_{2}\left(\alpha_{1}\right) \circ \Lambda_{1},
$$

with

$$
K_{t}^{\alpha_{t}}\left(\vec{\alpha}_{t-1}\right)=\sum_{i=1}^{r\left(\vec{\alpha}_{t-2}\right)}\left|\sigma_{\vec{\alpha}_{t}} \circ \min \left[r\left(\vec{\alpha}_{t-1}\right), i\right]\right\rangle\left\langle\left. i\right|_{W} \otimes E_{t}^{\alpha_{t}}\left(\vec{\alpha}_{t-1}, i\right),\right.
$$

where $\sigma_{\vec{\alpha}_{t}}$ is an injective map to the positive labels of a new incoherent basis (see footnote [62]); $E_{t}^{\alpha_{t}}\left(\vec{\alpha}_{t-1}, i\right)$ is an arbitrary operator acting on $Q$, potentially also depending on previous outcomes and controlled by the populations of the wire; and $1 \leq r\left(\vec{\alpha}_{t}\right)<r\left(\vec{\alpha}_{t-1}\right)$ for $t \geq 0$, with $r(0)=$ $\operatorname{dim}(W)$. Therefore, the length of the protocol $N$, possibly depending on the outcomes $\alpha_{t}$ one obtains, is bounded by $N \leq \operatorname{dim}(W)$.

One of the nontrivial results that we need to show in the proof of Proposition 3 is that classical to quantum forward can indeed be decomposed as described in the proposition. In the following lemma, we slightly generalize this statement, as it does not significantly complicate the proof and the statement might be of independent interest.
Lemma 11. Let $\Lambda=\sum_{\alpha} F^{\alpha} \cdot F^{\alpha \dagger}$ be a CPTP map acting on both $W$ and $Q$ with

$$
F^{\alpha}=\sum_{i=1}^{d}|f(i)\rangle\left\langle\left. i\right|_{W} \otimes E_{Q}^{\alpha}(i),\right.
$$

where $f$ maps indices to indices. Then $\Lambda$ admits a decomposition as in Proposition 3.

Proof.-For simplicity, we will only prove that there is a finite protocol of the given form. That the length of the protocol is bounded by the dimension of $W$ will be proven independently later in the proof of Proposition 3. The function $f$ in Eq. (A3) can map different members of the incoherent basis to the same output state. The main idea of the proof is to first reorder the incoherent basis of the wire (using a bijection) in such a way that we can then use a sequence of maps with Kraus operators of the form of Eq. (2) to implement the same map and the same subselection possibilities as with the given operators in Eq. (A3). The main trick is to iteratively collapse the subspaces belonging to the preimage of $|f(i)\rangle$.

Let us begin with the case that the image of $f(i)$ is $\{1, \ldots s\}$ for a $s \in \mathbb{N} \leq \operatorname{dim}(W)$. Define $W_{k}=\{i \mid f(i)=$ $k\}$ and a permutation $\sigma_{1}$ that maps the elements of $W_{k}$ to $\left\{1+\sum_{j=1}^{k-1}\left|W_{j}\right|, \ldots, \sum_{j=1}^{k}\left|W_{j}\right|\right\}$. This implements the announced reordering of the incoherent basis of the wire and corresponds to a unitary $\Lambda_{1}$ given by $K_{1}=\left|\sigma_{1}(i)\right\rangle\left\langle\left. i\right|_{W}\right.$. Next, we define $r_{t}=t+\sum_{j=1}^{s-t}\left|W_{j}\right|$, e.g., $r_{0}=\operatorname{dim}(W)$, $r_{1}=1+\sum_{j=1}^{s-1}\left|W_{j}\right|, \quad r_{s-1}=s-1+\left|W_{1}\right|, \quad$ and $\quad r_{s}=s$. With this, we then define (for $t \in\{2, \ldots s+1\}$ )

$$
\begin{aligned}
& K_{t}^{\alpha_{t}}=\sum_{i=1}^{r_{t-2}}\left|\sigma_{\oplus}^{r_{t-1}} \circ \min \left[r_{t-1}, i\right]\right\rangle\left\langle\left. i\right|_{W}\right. \\
& \otimes\left\{\begin{array}{ll}
E_{Q_{1}}^{\alpha_{t}}(i) \otimes\left|\alpha_{t}\right\rangle_{Q_{2}}, & i \geq r_{t-1} \\
\mathbb{1}_{Q_{1}}, & i<r_{t-1}
\end{array},\right.
\end{aligned}
$$

where the permutation $\sigma_{\oplus}^{l}$ is defined by the mapping $i \mapsto i+1$, for $i<l$ and $l \mapsto 1$. These Kraus operators are of the form given in Eq. (2).

From the CPTP condition for the Kraus operators defined in Eq. (A3), we get that $\sum_{i, j \in W_{k}}|i\rangle\left\langle\left. j\right|_{W} \otimes\right.$ $\sum_{\alpha} E_{Q}^{\alpha}(i)^{\dagger} E_{Q}^{\alpha}(j)=\sum_{i \in W_{k}}|i\rangle\left\langle\left. i\right|_{W} \otimes \sum_{\alpha} E_{Q}^{\alpha}(i)^{\dagger} E_{Q}^{\alpha}(i)=\mathbb{1}\right.$.

This implies that, for each $t \in\{2, \ldots s+1\}, K_{t}^{\alpha_{t}}$ are the Kraus operators of a CPTP map, that is, $\sum_{\alpha_{t}} K_{t}^{\alpha_{t} \dagger} K_{t}^{\alpha_{t}}=\mathbb{1}$.

It is straightforward to see by induction that

$$
\begin{aligned}
K_{t}^{\alpha_{t}} \circ \ldots \circ K_{1}= & \sum_{i \in W_{s-t+2}}|1\rangle\left\langle\left. i\right|_{W} \otimes E_{Q_{1}}^{\alpha_{t}}(i) \otimes \mid \alpha_{t}\right\rangle_{Q_{2}}+\sum_{i \in W_{s-t+3}}|2\rangle\left\langle\left. i\right|_{W} \otimes E_{Q_{1}}^{\alpha_{t-1}}(i) \otimes \mid \alpha_{t-1}\right\rangle_{Q_{2}}+\ldots \\
& +\sum_{i \in W_{s}}|t-1\rangle\left\langle\left. i\right|_{W} \otimes E_{Q_{1}}^{\alpha_{2}}(i) \otimes \mid \alpha_{2}\right\rangle_{Q_{2}}+\sum_{i \in \bigcup_{j=1}^{s-t+1} W_{s}}\left|\sigma_{1}(i)\right\rangle\left\langle\left. i\right|_{W} \otimes \mathbb{1}_{Q}\right. \\
= & \sum_{u=2}^{t} \sum_{i \in W_{s-t+u}}|u-1\rangle\left\langle\left. i\right|_{W} \otimes E_{Q_{1}}^{\alpha_{t+2-u}}(i) \otimes \mid \alpha_{t+2-u}\right\rangle+\sum_{i \in \bigcup_{j=1}^{s-t+1} W_{s}}\left|\sigma_{1}(i)\right\rangle\left\langle\left. i\right|_{W} \otimes \mathbb{1}_{Q} .\right.
\end{aligned}
$$


Now, we redefine the map $\Lambda_{s+1} \rightarrow \operatorname{Tr}_{Q_{2}} \Lambda_{s+1}$, from which follows the statement in the case that the image of $f(i)$ is $\{1, \ldots s\}$ for a $s \in \mathbb{N} \leq \operatorname{dim}(W)$. Now, assume that the image of $f$ is not $\{1, \ldots s\}$. In this case, we can proceed in the same way and change the permutation of $\Lambda_{s+1}$ (before we trace out $Q_{2}$ ) such that we implement the correct $f$. $\square$

We are now ready to give the proof of Proposition 3.

Proof of Proposition 3.-We need to prove four statements:

(1) Any elemental LOP map can be decomposed into an arbitrarily long sequence of CPTP maps represented by Kraus operators of the form given in Eq. (2).

(2) Any CPTP map given by Kraus operators as in Eq. (2) can be decomposed as a LOP map.

(3) Induction +1 : The composition of two CPTP maps that can be decomposed into an arbitrarily long sequence of CPTP maps represented by Kraus operators of the form given in Eq. (2) can again be decomposed into this form. This statement is trivial.

(4) Any CPTP map that can be decomposed into an arbitrarily long sequence of CPTP maps represented by Kraus operators of the form given in Eq. (2) can also be decomposed into such a sequence with $r\left(\vec{\alpha}_{t+1}\right)<r\left(\vec{\alpha}_{t}\right)$. From this follows that the choice $N \leq \operatorname{dim}(W)$ is always possible, since $r(0)$ is without loss of generality equal to $\operatorname{dim} W$.

The first statement is easy to see; permutations of the basis $\mathcal{Z}$ of $W$, diagonal unitaries on $W$, and observed quantum operations on $Q$ all have the form of $K_{1}^{\alpha_{1}}$. That classical to quantum forwarding has the form (2) is a direct corollary of Lemma 11.

To implement a map given by Kraus operators of the form (2) $\left[K_{t}^{\alpha_{t}}\left(\vec{\alpha}_{t-1}\right)=\sum_{i=1}^{r\left(\vec{\alpha}_{t-2}\right)}\left|\sigma_{\vec{\alpha}_{t}} \circ \min \left[r\left(\vec{\alpha}_{t-1}\right), i\right]\right\rangle\left\langle\left. i\right|_{W} \otimes\right.\right.$ $\left.E_{t}^{\alpha_{t}}\left(\vec{\alpha}_{t-1}, i\right)\right]$ by elemental operations, the first step is to implement the trivial observed quantum operation

$$
|0\rangle_{W_{2}},
$$

followed by a permutation on $W$ given by

$$
\sum_{i=1}^{r\left(\vec{\alpha}_{t-2}\right)}\left|\min \left[r\left(\vec{\alpha}_{t-1}\right), i\right]\right\rangle\left\langle\left. i\right|_{W_{1}} \otimes \mid i\right\rangle\left\langle\left. 0\right|_{W_{2}} .\right.
$$

Then we do a classical to quantum forwarding of system $W_{2}$ to an ancillary system $Q_{2}$. Up to here, we can summarize the concatenation of these operations by

$$
\sum_{i=1}^{r\left(\vec{\alpha}_{t-2}\right)}\left|\min \left[r\left(\vec{\alpha}_{t-1}\right), i\right]\right\rangle\left\langle\left. i\right|_{W_{1}} \otimes \mathbb{1}_{Q_{1}} \otimes \mid i\right\rangle_{Q_{2}} .
$$

The next step is a quantum operation on $Q$ defined by the Kraus operators,

$$
\sum_{i=1}^{r\left(\vec{\alpha}_{t-2}\right)} E_{t}^{\alpha_{t}}\left(\vec{\alpha}_{t-1}, i\right)_{Q_{1}} \otimes\left|\sigma_{\vec{\alpha}_{t}} \circ \min \left[r\left(\vec{\alpha}_{t-1}\right), i\right]\right\rangle\left\langle\left. i\right|_{Q_{2}},\right.
$$

which is a quantum operation exactly if the Kraus operators $K_{t}^{\alpha_{t}}\left(\vec{\alpha}_{t-1}\right)$ form one. In total, we then have

$$
\begin{aligned}
& \sum_{i=1}^{r\left(\vec{\alpha}_{t-2}\right)}\left|\min \left[r\left(\vec{\alpha}_{t-1}\right), i\right]\right\rangle\left\langle\left. i\right|_{W_{1}}\right. \\
& \quad \otimes E_{t}^{\alpha_{t}}\left(\vec{\alpha}_{t-1}, i\right)_{Q_{1}} \otimes\left|\sigma_{\vec{\alpha}_{t}} \circ \min \left[r\left(\vec{\alpha}_{t-1}\right), i\right]\right\rangle_{Q_{2}} .
\end{aligned}
$$

After a permutation on $W_{1}$ that implements $\sigma_{\vec{\alpha}_{t}}$, we obtain, in total,

$$
\begin{aligned}
& \sum_{i=1}^{r\left(\vec{\alpha}_{t-2}\right)}\left|\sigma_{\vec{\alpha}_{t}} \circ \min \left[r\left(\vec{\alpha}_{t-1}\right), i\right]\right\rangle\left\langle\left. i\right|_{W_{1}} \otimes E_{t}^{\alpha_{t}}\left(\vec{\alpha}_{t-1}, i\right)_{Q_{1}}\right. \\
& \quad \otimes\left|\sigma_{\vec{\alpha}_{t}} \circ \min \left[r\left(\vec{\alpha}_{t-1}\right), i\right]\right\rangle_{Q_{2}} .
\end{aligned}
$$

The last step is to use the operation $\mathcal{B}_{W_{1}, Q_{2}}^{-1}$ from Lemma 2 to get rid of $Q_{2}$, and we end up with the wanted operation.

As already mentioned, the third statement is trivial. The hard part is the fourth statement. Assume we have two arbitrary sets of Kraus operators $K_{t}^{\alpha_{t}}, K_{t-1}^{\alpha_{t-1}}$ of the given form corresponding to $\Lambda_{t}$ and $\Lambda_{t-1}$. Then, we distinguish two cases. In the first case, we show that we can find two new sets of Kraus operators $L_{t}^{\alpha_{t}}, L_{t-1}^{\alpha_{t-1}}$ of the required form such that $K_{t}^{\alpha_{t}} \circ K_{t-1}^{\alpha_{t-1}}=L_{t}^{\alpha_{t}} \circ L_{t-1}^{\alpha_{t-1}}, r\left(\vec{\alpha}_{t-2}\right)$ remains the same, and $r\left(\vec{\alpha}_{t-1}\right)<r\left(\vec{\alpha}_{t-2}\right)$ for the two new sets [the place where one cuts the Hilbert space dimension of the wire in the step $t\left[r\left(\vec{\alpha}_{t-1}\right)\right]$ depends on the previous outcomes, but not on the current one, which is why its index is $t-1$ and not $t]$. In the second case, one can replace the two CPTP maps by one CPTP map of the required form such that $r\left(\alpha_{t-2}\right)$ remains unchanged.

Assume $r\left(\vec{\alpha}_{t-1}\right) \geq r\left(\vec{\alpha}_{t-2}\right)$ (otherwise, there is nothing to show). First, we split up the injection $\sigma_{\vec{\alpha}_{t-1}}(i)$ into a permutation and an order-preserving injection. Formally, we define the permutation $\eta_{\vec{\alpha}_{t-1}}$ on $\left\{1, \ldots r\left(\vec{\alpha}_{t-2}\right)\right\}$ and the injection $a:\left\{1, \ldots r\left(\vec{\alpha}_{t-2}\right)\right\} \rightarrow \sigma_{\vec{\alpha}_{t-1}}\left(\left\{1, \ldots r\left(\vec{\alpha}_{t-2}\right)\right\}\right) \subset$ $\mathbb{N}^{>0}$, such that

$$
\sigma_{\vec{\alpha}_{t-1}}(i)=a\left[\eta_{\vec{\alpha}_{t-1}}(i)\right],
$$

and $a(i)<a(j)$ for $i<j$. Then, there is some $l \leq r\left(\vec{\alpha}_{t-2}\right)$, such that

$$
\min \left[r\left(\vec{\alpha}_{t-1}\right), \cdot\right] \circ \sigma_{\vec{\alpha}_{t-1}}\left(\left\{1, \ldots r\left(\vec{\alpha}_{t-2}\right)\right\}\right)=\{a(1), \ldots a(l)\}
$$

and

$$
\begin{aligned}
& \min \left[r\left(\vec{\alpha}_{t-1}\right), \cdot\right] \circ \sigma_{\vec{\alpha}_{t-1}} \\
& \quad=\min \left[r\left(\vec{\alpha}_{t-1}\right), \cdot\right] \circ a \circ \eta_{\vec{\alpha}_{t-1}}=a \circ \min [l, \cdot] \circ \eta_{\vec{\alpha}_{t-1}},
\end{aligned}
$$


with $f(x, \cdot)$ denoting the function $f(x, y)$ for fixed $x$, as a function of $y$, and we use $f(A)$ for a function $f$ and a set $A$ to denote the image of the set $A$ under $f$.

We first consider the case $l<r\left(\vec{\alpha}_{t-2}\right)$. We first define

$$
F_{t}^{\alpha_{t}}\left(\vec{\alpha}_{t-1}, i\right)=E_{t}^{\alpha_{t}}\left[\vec{\alpha}_{t-1}, \sigma_{\vec{\alpha}_{t-1}} \circ \eta_{\vec{\alpha}_{t-1}}^{-1}(i)\right]=E_{t}^{\alpha_{t+1}}\left[\vec{\alpha}_{t}, a(i)\right],
$$

with $i \in\left\{1, \ldots r\left(\vec{\alpha}_{t-2}\right)\right\}$. By further defining the injection $\eta_{\vec{\alpha}_{t}}(k)=\sigma_{\vec{\alpha}_{t}}[a(k)]$, we can finally define

$$
L_{t}^{\alpha_{t}}\left(\vec{\alpha}_{t-1}\right)=\sum_{i=1}^{r\left(\vec{\alpha}_{t-2}\right)}\left|\eta_{\vec{\alpha}_{t}} \circ \min [l, i]\right\rangle\left\langle\left. i\right|_{W} \otimes F_{t}^{\alpha_{t}}\left(\vec{\alpha}_{t-1}, i\right)\right.
$$

and

$$
\begin{aligned}
& L_{t-1}^{\alpha_{t-1}}\left(\vec{\alpha}_{t-2}\right) \\
& \quad=\sum_{i=1}^{r\left(\vec{\alpha}_{t-3}\right)}\left|\eta_{\vec{\alpha}_{t-1}} \circ \min \left[r\left(\vec{\alpha}_{t-2}\right), i\right]\right\rangle\left\langle\left. i\right|_{W} \otimes E_{t-1}^{\alpha_{t-1}}\left(\vec{\alpha}_{t-2}, i\right) .\right.
\end{aligned}
$$

First, we note that the map defined by $L_{t-1}^{\alpha_{t-1}}\left(\vec{\alpha}_{t-2}\right)$ is CPTP exactly if the map defined by $K_{t-1}^{\alpha_{t-1}}\left(\vec{\alpha}_{t-2}\right)$ is (as they only differ by a permutation at the end).

Now, we show that $L_{t}^{\alpha_{t}}\left(\vec{\alpha}_{t-1}\right)$ forms a CPTP map as well. Remember that $\min \left[r\left(\vec{\alpha}_{t-1}\right), a(\cdot)\right]=a(\min [l, \cdot])$ and, since $a$ is a bijection on its image, $\left\langle\min \left[r\left(\vec{\alpha}_{t}\right), a(i)\right]\right| \min \left[r\left(\vec{\alpha}_{t}\right)\right.$, $a(j)]\rangle=\langle\min [l, i] \mid \min [l, j]\rangle$. Then,

$$
\begin{aligned}
& \mathbb{1}=\sum_{\alpha_{t}} K_{t}^{\alpha_{t}}\left(\vec{\alpha}_{t-1}\right)^{\dagger} K_{t}^{\alpha_{t}}\left(\vec{\alpha}_{t-1}\right) \\
& \Leftrightarrow \forall i, j: \\
& \mathbb{1} \delta_{i, j}=\left(\left\langle\left. i\right|_{W} \otimes \mathbb{1} Q\right)\left(\sum_{\alpha_{t}} K_{t}^{\alpha_{t}}\left(\vec{\alpha}_{t-1}\right)^{\dagger} K_{t}^{\alpha_{t}}\left(\vec{\alpha}_{t-1}\right)\right)\left(|j\rangle_{W} \otimes \mathbb{1}_{Q}\right)\right. \\
&=\sum_{\alpha_{t}}\left\langle\min \left[r\left(\vec{\alpha}_{t-1}\right), i\right] \mid \min \left[r\left(\vec{\alpha}_{t-1}\right), j\right]\right\rangle E_{t}^{\alpha_{t}}\left(\vec{\alpha}_{t-1}, i\right)^{\dagger} E_{t}^{\alpha_{t}}\left(\vec{\alpha}_{t-1}, j\right) \\
& \Leftrightarrow \forall i, j: \\
& \mathbb{1} \delta_{i, j}=\sum_{\alpha_{t}}\left\langle\min \left[r\left(\vec{\alpha}_{t}\right), a(i)\right] \mid \min \left[r\left(\vec{\alpha}_{t}\right), a(j)\right]\right\rangle \\
& E_{t}^{\alpha_{t}}\left(\vec{\alpha}_{t-1}, a(i)\right)^{\dagger} E_{t}^{\alpha_{t}}\left(\vec{\alpha}_{t-1}, a(j)\right) \\
&=\sum_{\alpha_{t}}\langle\min [l, i] \mid \min [l, j]\rangle F_{t}^{\alpha_{t}}\left(\vec{\alpha}_{t-1}, i\right)^{\dagger} F_{t}^{\alpha_{t}}\left(\vec{\alpha}_{t-1}, j\right) \\
& \Leftrightarrow \mathbb{1}=\sum_{\alpha_{t}} L_{t}^{\alpha_{t}}\left(\vec{\alpha}_{t-1}\right)^{\dagger} L_{t}^{\alpha_{t}}\left(\vec{\alpha}_{t-1}\right),
\end{aligned}
$$

where we used in the last line that $\eta_{\vec{\alpha}_{t}}$ is a bijection as well.

Finally, we need to check that we get, indeed, the right mapping $\left[K_{t}^{\alpha_{t}}\left(\vec{\alpha}_{t-1}\right) \circ K_{t-1}^{\alpha_{t-1}}\left(\vec{\alpha}_{t-2}\right)=L_{t}^{\alpha_{t}}\left(\vec{\alpha}_{t-1}\right) \circ L_{t-1}^{\alpha_{t-1}}\left(\vec{\alpha}_{t-2}\right)\right]$. This follows from the equalities

$$
\begin{aligned}
& \sum_{i=1}^{r\left(\vec{\alpha}_{t-2}\right)}\left|\sigma_{\vec{\alpha}_{t}} \circ \min \left[r\left(\vec{\alpha}_{t-1}\right), i\right]\right\rangle\left\langle i|\circ| \sigma_{\vec{\alpha}_{t-1}}(j)\right\rangle \otimes E_{t}^{\alpha_{t}}\left(\vec{\alpha}_{t-1}, i\right) \circ E_{t-1}^{\alpha_{t-1}}\left(\vec{\alpha}_{t-2}, j\right) \\
& \quad=\left|\sigma_{\vec{\alpha}_{t}} \circ \min \left[r\left(\vec{\alpha}_{t-1}\right), \sigma_{\vec{\alpha}_{t-1}}(j)\right]\right\rangle \otimes E_{t}^{\alpha_{t}}\left(\vec{\alpha}_{t-1}, \sigma_{\vec{\alpha}_{t-1}}(j)\right) \circ E_{t-1}^{\alpha_{t-1}\left(\vec{\alpha}_{t-2}, j\right)} \\
& =\left|\sigma_{\vec{\alpha}_{t}}^{\circ} \circ \circ \min \left[l, \eta_{\vec{\alpha}_{t}}(j)\right]\right\rangle \otimes E_{t}^{\alpha_{t}}\left(\vec{\alpha}_{t-1}, a\left(\eta_{\vec{\alpha}_{t-1}}(j)\right)\right) \circ E_{t-1}^{\alpha_{t-1}\left(\vec{\alpha}_{t-2}, j\right)} \\
& \left.=\left|\eta_{\vec{\alpha}_{t}} \circ \min \left[l, \eta_{\vec{\alpha}_{t-1}}(j)\right]\right\rangle \otimes F_{t}^{\alpha_{t}}\left(\vec{\alpha}_{t-1}, \eta_{\vec{\alpha}_{t-1}}(j)\right)\right) \circ E_{t-1}^{\alpha_{t-1}\left(\vec{\alpha}_{t-2}, j\right)} \\
& \left.=\sum_{i=1}^{r\left(\vec{\alpha}_{t-2}\right)}\left|\eta_{\vec{\alpha}_{t}} \circ \min [l, i]\right\rangle\left\langle i|\circ| \eta_{\vec{\alpha}_{t-1}}(j)\right\rangle \otimes F_{t}^{\alpha_{t}}\left(\vec{\alpha}_{t-1}, i\right)\right) \circ E_{t-1}^{\alpha_{t-1}\left(\vec{\alpha}_{t-2}, j\right) .}
\end{aligned}
$$

The case $l=r\left(\vec{\alpha}_{t-1}\right)$ can be handled similarly, just by noting that the action of $\min [l, \cdot]$ gets trivial, and one can, therefore, express the concatenation of the two maps as a single map of the same form. 
This proves that one can chose $r$ such that $\operatorname{dim} W \geq r(0)>r\left(\vec{\alpha}_{1}\right)>\ldots>r\left(\vec{\alpha}_{N-1}\right) \geq 1$. It follows that it is always possible to have $N \leq \operatorname{dim} W$.

We can now use the above proposition to prove one direction of the connection between LOP and SIO operations given in Proposition 4.

Proposition (4). Let $\Lambda$ be a CPTP map acting on $W \otimes Q . \Lambda$ is a LOP operation exactly if it can be written as a sequence of maps $\Lambda=\Lambda_{M}\left(\vec{\alpha}_{M-1}\right) \circ \ldots \circ \Lambda_{1}$, for some finite $M$, where each $\Lambda_{i}$ is

(a) a physical incoherent operation (see Ref. [40]) on $W$ or

(b) a destructive measurement in one fully coherent basis of a subsystem of $W$ or

(c) a controlled unitary $\left[\mathbf{U}_{\text {control }}=\sum_{m}|m\rangle\left\langle\left. m\right|_{\text {control }} \otimes\right.\right.$ $\mathbf{U}_{\text {target }}(m)$ ] with control $W$ and target $Q$ or

(d) a generalized measurement of $Q$, encoding the result on $W\left(\rho \mapsto \sum_{\alpha}|\alpha\rangle\left\langle\left.\alpha\right|_{W} \otimes K^{\alpha} \rho K^{\alpha \dagger}\right)\right.$.

One can equivalently replace item (a) by "a strictly incoherent operation on $W$."

Proof.-We start the proof by noting that any destructive measurements on a subsystem of $W$ can be done by LOP operations by classical to quantum forwarding of the subsystem in question to an ancilla in $Q, Q_{2}$, and then doing the measurement there. Strictly incoherent operations are those with a Kraus decomposition, with Kraus operators of the form $K^{\alpha_{1}}=\sum_{i=1}^{d} c(\alpha)\left|\sigma_{\alpha}(i)\right\rangle\langle i|$ [24], which obviously is a special case of the form $K_{1}$ in Proposition 3 [i.e., Eq. (2)]; the same is true for control unitaries of the form (c). Next, we note that PIO is a subset of SIO [40], so that any operation having a decomposition as in the proposition is an element of LOP.

For the converse, we only need to show that we can do classical to quantum forwarding using only the operations (a)-(d). This goes by virtually the same protocol we already applied for the inverse part of Lemma 2. We first do a controlled unitary from the system $W_{s}$ in question to an ancillary system $Q_{s}$ prepared in the state $|0\rangle$, to which we want to teleport and apply a measurement in the Fourier basis of $W_{s}$ (note that measurements in different fully coherent bases only differ by a diagonal unitary, which is an element of PIO, so we can assume without loss of generality that the basis we can measure in is given by the Fourier basis), followed by a correction of the phase on $Q_{s}$ :

(1) $|0\rangle_{Q}$

(2) $\sum_{i}|i\rangle\left\langle\left. i\right|_{W_{s}} \otimes \sum_{j} \mid i \oplus j\right\rangle\left\langle\left. j\right|_{W_{s}}\right.$

(3) $F T_{W_{s}}^{k}=\left\langle\left.\hat{k}\right|_{W_{s}}=\sum_{j=1}^{\operatorname{dim}\left(W_{s}\right)}\left[\left(e^{2 \pi i k j / \operatorname{dim}\left(W_{s}\right)}\right) /\right.\right.$ $\left.\sqrt{\operatorname{dim}\left(W_{s}\right)}\right]\left\langle\left. j\right|_{W_{s}}\right.$

(4) $D(k)=\sum_{l=1}^{\operatorname{dim}\left(W_{s}\right)}|l\rangle\left\langle\left. l\right|_{Q_{s}} e^{-2 \pi i k l / \operatorname{dim}\left(W_{s}\right)}\right.$, resulting in the action $\sum_{i}\left\langle\left. i\right|_{W_{s}} ^{Q_{s}} \otimes \mid i\right\rangle_{Q_{s}}$

The most general set of meaningful operations, if one just has the restriction that one wants to keep the wire incoherent and classically correlated with the quantum system, is the operations that do not allow us to generate states that are not incoherent quantum from those that are. If one allows postselection, this means that the same should be true for each Kraus operator defining the operation. This set, introduced in Ref. [42], is called incoherent-quantum operations (IQO). Our approach, by contrast, is operational, in the sense that we only allow some specific elemental operations that are meaningful. This has the advantage that it is more transparent and does not allow for operations that could be nonclassical in a way that might not be obvious, but the disadvantage is that there might be some operations that we do not allow that are still meaningful. Fortunately, we can prove that the gap between the theory we propose and the completely abstractly defined maximal possible theory IQO is not that big. The details of this statement are the content of Proposition 6.

Proposition (6). Be $\Lambda$ an incoherent-quantum operation on $W_{1} \otimes Q$, which is exactly the case if it is CPTP and has a Kraus decomposition with Kraus operators of the form $K^{\alpha}=\sum_{i}\left|f_{\alpha}(i)\right\rangle\left\langle\left. i\right|_{W_{1}} \otimes E^{\alpha}(i)\right.$, for some functions $f_{\alpha}$ acting on the labels of the incoherent basis and some operators $E^{\alpha}(i)$ acting on $Q$. Let $d:=\operatorname{dim}\left(W_{1}\right)$. If $d=2, \Lambda \in$ LOP. For $d \geq 3$, LOP $\neq \mathrm{IQO}$, but there is a stochastic implementation of the map in LOP with a success rate of at least $1 / d$; i.e., there is an operation $\Lambda^{\prime} \in \operatorname{LOP}\left(W_{2} \otimes W_{1} \rightsquigarrow Q\right)$ with $\Lambda^{\prime}\left[|0\rangle\left\langle\left. 0\right|_{W_{2}} \otimes \rho\right]=|0\rangle\left\langle\left. 0\right|_{W_{2}} \otimes \Lambda_{0}^{\prime}[\rho]+\mid 1\right\rangle\left\langle\left. 1\right|_{W_{2}} \otimes\right.\right.$ $\Lambda_{1}{ }^{\prime}[\rho]$ with $\Lambda_{1}{ }^{\prime}[\rho]=\Lambda[\rho] / d \forall \rho$.

Proof.-The form of the incoherent-quantum operations directly follows from applying each Kraus operator to a product state incoherent on $W$ and requiring that it is still (up to normalization) a product state incoherent on $W$. The converse, namely, that any Kraus operator of that form is incoherent quantum (that is, preserves the set of incoherentquantum states), is a trivial consequence of the convexity of the set of incoherent-quantum states.

The protocol for doing the operation given by the Kraus operators $K^{\alpha}=\sum_{i}\left|f_{\alpha}(i)\right\rangle\left\langle\left. i\right|_{W} \otimes E_{\alpha}(i)_{Q}\right.$, by LOP operations with a probability of $1 / d$ is given by (identifying $W=W_{1}$ and $Q=Q_{1}$ at the beginning and the end)

(1) $\sum_{i}|i\rangle\left\langle\left. i\right|_{W_{1}} \otimes \mid i\right\rangle_{Q_{2}}$ (see Lemma 2),

(2) $\sum_{i} E_{\alpha}(i)_{Q_{1}} \otimes\left|f_{\alpha}(i)\right\rangle\left\langle\left. i\right|_{Q_{2}}\right.$,

(3) $\sum_{i}\left|f_{\alpha}(i)\right\rangle\left\langle\left. i\right|_{W_{1}} \otimes \mid i\right\rangle_{W_{2}}$ (a permutation after adding an ancilla),

(4) "Delete" the duplicate $Q_{2}$ (applying $\mathcal{B}^{-1}$ of Lemma 2 to $W_{1}, Q_{2}$ ),

(5) $\sum_{i}\left\langle\left. i\right|_{W_{2}} \otimes \mid i\right\rangle_{Q_{2}}$,

(6) $\left\langle\left.\hat{k}\right|_{Q_{2}}\right.$.

In total, the operation is given by the Kraus operators $\sum_{i}\left|f_{\alpha}(i)\right\rangle\left\langle\left. i\right|_{W} \otimes E^{\alpha}(i)_{Q} \cdot\langle\hat{k} \mid i\rangle\right.$. If the outcome is $k=0$, $\langle\hat{k} \mid i\rangle=1 / \sqrt{d} \forall i$ and the protocol is successful. Note that the probability for this is $1 / d$, independently of the initial state the operation is applied to. If $k \neq 0$, there is an $i$-dependent phase and, in general, the protocol fails (the information about $i$ is lost, so that, at this point, there is no way to correct the phases). 
Let us consider the case $d=2$. We define the set $R=$ $\left\{\alpha \mid f_{\alpha}(1)=f_{\alpha}(2)\right\}$ and $R^{c}$ its complement, separating the injective from the noninjective functions on $W$. The idea in the following is to first check (on $Q$ ) whether one has an injective or a noninjective case on $W$ and then change the form on $W$ accordingly, while inverting the check and applying the final operation on $Q$. Let without loss of generality $\alpha \in\{1, \ldots N\}$. We note that, since the $K^{\alpha}$ form a CPTP map, we have, in particular, that $\sum_{\alpha \in R} E^{\alpha}(1)_{Q^{\circ}}^{\dagger} E^{\alpha}(2)_{Q}=0$. For this reason, it makes sense to define the operations $E^{0}(i)=\sqrt{\sum_{\alpha \in R} E^{\alpha}(i)_{Q}^{\dagger} \circ E^{\alpha}(i)_{Q}}$ and $K^{0}=\sum_{i}|i\rangle\langle i| \otimes E^{0}(i)$, and it is easy to check that the $K^{\alpha}$, with $\alpha \in\{0\} \cup R^{c}$, again form a CPTP map. This map has the form of Eq. (2) and is therefore an element of LOP.
For $\alpha \in R$, we then need a second step, and we define the operations $E_{1}^{\alpha}(i)=E^{\alpha}(i) \circ E^{0}(i)^{-1}$, where $E^{0}(i)^{-1}$ is the Penrose pseudoinverse of $E^{0}(i)$; that is, if $E^{0}(i)=$ $U(i) \circ D(i) \circ U(i)^{\dagger}$ is the singular value decomposition of $E^{0}(i)$ [where we used that $E^{0}(i)=E^{0}(i)^{\dagger}$, from the definition of $\left.E^{0}(i)\right]$, then $E^{0}(i)^{-1}=U(i) \circ D(i)^{-1} \circ U(i)^{\dagger}$ [here, $D(i)$ is a diagonal matrix, and $D(i)^{-1}$ is its diagonal right inverse on its support, and vice versa]. We also need the operation $E_{1}^{0}(i)=|i\rangle \otimes\left[\mathbb{1}-E^{0}(i) \circ E^{0}(i)^{-1}\right]$. Here, it is useful to note that $E^{0}(i) \circ E^{0}(i)^{-1}=E^{0}(i)^{-1} \circ E^{0}(i)$ is the projection on the image of $E^{0}(i)$. We can then define, in the notation of Proposition 3, $K_{1}^{\alpha}=\sum_{i}|1\rangle\langle i| \otimes E_{1}^{\alpha}(i)$ for $\alpha \in$ $R \cup\{0\}\left[f_{1}(i)=1\right]$. We then have that

$$
\begin{aligned}
\sum_{\alpha} K_{1}^{\alpha \dagger} K_{1}^{\alpha} & =\sum_{\alpha} \sum_{i, j}|i\rangle\langle j| \otimes E_{1}^{\alpha}(i)^{\dagger} E_{1}^{\alpha}(j) \\
& =\sum_{i, j}|i\rangle\left\langle j|\otimes E^{0}(i)^{-1} \overbrace{\sum_{\alpha \in R}\left(E^{\alpha}(i)^{\dagger} E^{\alpha}(j)\right)}^{\delta_{i, j} E^{0}(i)^{2}} E^{0}(j)^{-1}+\sum_{i, j}| i\right\rangle\langle j| \otimes\langle i \mid j\rangle\left(\mathbb{1}-E^{0}(i) \circ E^{0}(i)^{-1}\right)^{\dagger}\left(\mathbb{1}-E^{0}(i) \circ E^{0}(i)^{-1}\right) \\
& =\sum_{i}|i\rangle\left\langle i\left|\otimes E^{0}(i)^{-1} E^{0}(i)+\sum_{i}\right| i\right\rangle\langle i| \otimes\left(\mathbb{1}-2 E^{0}(i) E^{0}(i)^{-1}+E^{0}(i) E^{0}(i)^{-1}\right)=\mathbb{1}
\end{aligned}
$$

Noticing that the probability to measure $\alpha=0$ in the second step, provided that the outcome in the first was $\alpha=0$, is 0 , and that $E^{\alpha}(i) \circ E^{0}(i)^{-1} \circ E^{0}(i)=E^{\alpha}(i)$ [since the support of $E^{0}(i)$ contains the support of $E^{\alpha}(i)$ ], we find that, indeed, applying the protocol as in Proposition 3 with the above defined operations yields the right map.

The proof of the statement LOP $\neq \mathrm{IQO}$ is done in Appendix F, where we provide an explicit counterexample for a wire with Hilbert-space dimension 3.

Whether one can meaningfully call states that are not free in a given resource theory "resources" depends on whether they can be used to do tasks that are impossible under the application of free operations alone. Theorem 5 shows that maximally coherent states are resources in the maximal sense of the word: They enable us to do anything within quantum mechanics.

Theorem (5). Let $\Lambda$ be a CPTP map acting on $W_{1} \otimes Q$, with $W_{1}$ having dimension $d$. Let $|\psi\rangle=\sum_{i=1}^{d}(1 / \sqrt{d})|i\rangle$ be a maximally coherent state on $W_{2}$. Then, there is an operation $\Lambda^{\prime} \in \operatorname{LOP}\left(W_{2} \otimes W_{1} \lll Q\right)$, with $\operatorname{Tr}_{W_{2}}\left[\Lambda^{\prime}\left[|\psi\rangle\left\langle\left.\psi\right|_{W_{2}} \otimes\right.\right.\right.$ $\left.\left.\rho_{W_{1}, Q}\right]\right]=\Lambda\left[\rho_{W_{1}, Q}\right]$.

Proof.-The trick is to do the operation on the quantum side; that is, send the system $W_{1}$ to $Q_{2}$ and do the operation
$\Lambda$ on $Q_{2}, Q_{1}$. Then use Lemma 2 to construct a Bell-type state from the ancillary coherent state on $W_{2}$. Finally, teleport the system $Q_{2}$ back to $W_{1}$ using the original teleportation protocol [73] and using up the ancillary Bell state. In detail (identifying $Q_{1}=Q$ at the beginning and at the end),

(1) Preparation: $|\psi\rangle_{W_{2}}$,

(2) (Free) teleportation to the quantum side $\sum_{i}\left\langle\left. i\right|_{W_{1}} \otimes\right.$ $|i\rangle_{Q_{2}}$,

(3) Application of $\Lambda$ on the quantum side: $\Lambda_{Q_{2}, Q_{1}}$,

(4) Doubling of $W_{2}$ (Lemma 2): $\sum_{i}|i\rangle\left\langle\left. i\right|_{W_{2}} \otimes \mid i\right\rangle_{Q_{3}}$,

(5) Measurement in the "Bell basis" $\left\langle\left. b(k, l)\right|_{Q_{3}, Q_{2}}\right.$, given by $|b(k, l)\rangle=\mathrm{CNOT \circ}(|\hat{k}\rangle \otimes|l\rangle)=1 / \sqrt{d}$ $\sum_{j} e^{2 \pi i k j / d}|j\rangle \otimes|l \oplus(j-1)\rangle$, with $\quad a \oplus b=$ $\bmod _{d}(a+b-1)+1$,

(6) Finish with a diagonal unitary $\sum_{j} e^{2 \pi i k j / d}|j\rangle\left\langle\left. j\right|_{W_{2}}\right.$ on $W_{2}$ followed by a permutation $\sum_{j} \mid l \oplus(j-$ 1) $\rangle_{W_{1}}\left\langle\left. j\right|_{W_{2}}\right.$, both depending on the $d^{2}$ possible outcomes of the previous measurement, given by the indices $k, l$.

Using the Kraus decomposition for the map $\Lambda(\rho)=$ $\sum_{\alpha} K^{\alpha} \rho K^{\alpha \dagger}$, we get that the full protocol is given by the $\left(d^{2}\right)$ Kraus operators

$$
1 / d \sum_{i, j}|l \oplus(j-1)\rangle\left\langlei | _ { W _ { 1 } } \otimes \left(\left[\left\langle\left. l \oplus(j-1)\right|_{Q_{2}} \otimes \mathbb{1}_{Q_{1}}\right] \circ K^{\alpha} \circ\left(|i\rangle_{Q_{2}} \otimes \mathbb{1}_{Q_{1}}\right)\right)=K^{\alpha} / d\right.\right.
$$




\section{APPENDIX B: RELATION TO DIFFERENT CLASSES OF OPERATIONS}

In this section, we give additional details to the relation between LOP and some selected classes of operations discussed in the literature; see Fig. 4. A complete representation is beyond the scope of this article, but the interested reader can find more references to relevant articles about these and other classes in Ref. [25].

For coherence theory, the biggest classes of operations we want to mention are two natural classes whose action on the set of incoherent states can be modeled by stochastic operations and are closed under composition and mixing [74]. These are the ones that do not create coherence on average [the maximally incoherent operations (MIO) [22] ] and those that on average are independent of the initial coherence [coherence nonactivating operations (CNA) $[49,50]]$. The intersection of these two sets is the operations that can neither create nor detect coherence - the dephasing covariant operations (DIO) $[40,41,49]$. They are called so because they commute with dephasing. MIO contains a subset of operations that do not generate coherence even after postselection, the incoherent operations (IO) [23]. LOP ( $W \stackrel{\rightsquigarrow}{\longleftarrow}$ ) (meaning the effective coherence theory of LOP on the wire) is similar to IO, but not equivalent. LOP has a more complicated structure, but it is operationally motivated in the present article. Subsets of LOP are the SIO [24,39], which include translationally invariant operations (TIO) [41,51] inside the PIO [40]. Note that for the inclusion of TIO in PIO and SIO to be valid, the Hamiltonian of the system needs to be nondegenerate or, conversely, one needs to generalize coherence theory to a theory where the dephasing defining incoherent states is not total and leaves the degenerate subspaces of the Hamiltonian invariant (see Refs. [22,41]). These three theories (SIO, TIO, and PIO) also have operationally sensible interpretations (for instance, TIO play an important role in thermodynamics [75,76]). However, since SIO, TIO, and PIO are subsets of DIO, they cannot create or detect coherence (restricting the free POVMs to a very small set, namely, all those that cannot measure coherence). This means that coherence is not a useful resource for any task that is observable by free measurements in these sets.

For basis-dependent discord, we first have the class that leaves incoherent-quantum states invariant, even after postselection, the IQO [42]). Inside these (though it is still an open question whether this inclusion is strict), we have the general operations incoherent on $A$ (GOIA) [43] (here, $A=W$ ). These operations are very similar, but not equivalent, to $\operatorname{LOP}(W \rightsquigarrow Q)$. Instead of allowing permutations on the wire, in GOIA, any incoherent operation is allowed, making GOIA slightly less operationally meaningful. SIO [39], TIO [41], and PIO [40] can also be formulated as effective theories of basis-dependent discord theories, which, for convenience, we call by the same names. The connection between these extended versions of SIO, PIO, and $\operatorname{LOP}(W \rightsquigarrow Q)$ is given in the discussion of Proposition 4.
For entanglement between two distant parties, one has the set of separable operations (SEP) [48] that preserve separable states (states with no entanglement). Inside of these, there are the better operationally motivated, but more complex, LOCC $[48,77]$. This set is the same as the effective theory of LOP on the two parties, $\operatorname{LOP}\left(Q_{1} \stackrel{\leftrightarrow}{\rightarrow} 1 \stackrel{\rightsquigarrow}{\leftarrow} Q_{2}\right)$. Replacing the permutations on the wire in the basic definition of LOP by the identity or by incoherent operations, we still get the same effective theory, LOCC. However, in the proof of Theorem 7 (concerning the equivalence of the resources coherence and entanglement in the bipartite setting), we do need the permutations for one direction and, for the other, we do use that LOP only consists of the elemental operations presented here. Therefore, it is not clear for other sets of incoherent operations whether coherence on the wire and entanglement between the two parties are equivalent resources for bipartite tasks. Inside LOCC, finally, there are the operations that additionally to the locality restriction also ask that the local operations of the two parties be incoherent in a given basis, the local incoherent operations and classical communication (LICC) [29,30]. Replacing the incoherent operations there by $\operatorname{LOP}(W \stackrel{\rightsquigarrow}{\longleftarrow})$, we get

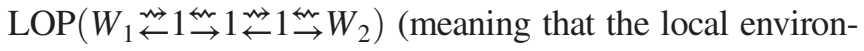
ment of the two parties is connected by a wire).

\section{APPENDIX C: PROOFS ON THE COHERENCE COST OF ENTANGLEMENT}

Both theorems on the coherence cost of entanglement presented in the main part depend heavily on Remark 9, which results from just following the respective protocols. To facilitate reading, we repeat it here:

Remark (9). $\forall \rho_{W}=\sum_{i} p_{i}|i\rangle\left\langle\left. i\right|_{W}\right.$,

$$
\begin{aligned}
& \text { 1. } \operatorname{Tr}_{W}\left(\Lambda \circ\left(\rho_{W} \otimes \mathbb{1}_{Q}\right)\right) \in \operatorname{LOCC}\left(Q^{1}, \ldots, Q^{n}\right)
\end{aligned}
$$

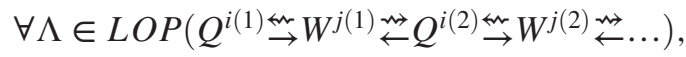

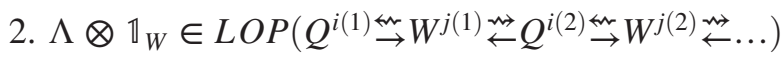

$$
\begin{aligned}
& \forall \Lambda \in \operatorname{LOCC}\left(Q^{1}, \ldots, Q^{n}\right) \text {. }
\end{aligned}
$$

We then have

Theorem (7). Let $\eta_{\text {LOCC }}=\sum_{i j} r_{i, j}|i\rangle\left\langle\left. j\right|_{Q_{1}^{1}} \otimes \mid i\right\rangle\left\langle\left. j\right|_{Q_{1}^{2}}\right.$ be a maximally correlated state (in arbitrary orthonormal local bases of $Q_{1}^{1} \otimes Q_{1}^{2}$ ) and $\eta_{\mathrm{LOP}}=\sum_{i j} r_{i, j}|i\rangle\left\langle\left. j\right|_{W}\right.$ be a corresponding state in the incoherent basis $\mathcal{Z}$ of $W$. If $\Lambda$ is a CPTP map on $Q^{1} \otimes Q^{2}$, then the following statements are equivalent:

$$
\begin{aligned}
\text { 1. } & \exists \Lambda_{\mathrm{LOCC}} \in \operatorname{LOCC}\left(Q^{1}, Q^{2}\right): \\
& \Lambda_{\mathrm{LOCC}}\left[\eta_{\mathrm{LOCC}} \otimes \rho_{Q_{2}^{1}, Q_{2}^{2}}\right]=\Lambda\left[\rho_{Q_{2}^{1}, Q_{2}^{2}}\right] \quad \forall \rho_{Q_{2}^{1}, Q_{2}^{2}} \\
\text { 2. } & \exists \Lambda_{\mathrm{LOP}} \in \operatorname{LOP}\left(Q^{1 \stackrel{\leftrightarrow}{\rightarrow}} W \stackrel{\text { ↔ }}{\leftarrow} Q^{2}\right): \\
& \Lambda_{\mathrm{LOP}}\left[\eta_{\mathrm{LOP}} \otimes \rho_{Q_{2}^{1}, Q_{2}^{2}}\right]=\Lambda\left[\rho_{Q_{2}^{1}, Q_{2}^{2}}\right] \quad \forall \rho_{Q_{2}^{1}, Q_{2}^{2}} .
\end{aligned}
$$


Proof.-The " $\Rightarrow$ " statement is a direct corollary of Remark 9 and Lemma 2. The operation $\mathcal{B}$ in Lemma 2 allows us to transform the state $\sum_{i j} r_{i, j}|i\rangle\left\langle\left. j\right|_{W} \otimes \rho_{Q_{2}^{1}, Q_{2}^{2}}\right.$ to $\sum_{i j} r_{i, j}|i i\rangle\left\langle\left. j j\right|_{W, Q_{2}^{2}} \otimes \rho_{Q_{2}^{1}, Q_{2}^{2}}\right.$, which by classical to quantum forwarding can in turn be transformed to $\sum_{i j} r_{i, j}|i i\rangle\left\langle\left. j j\right|_{Q_{2}^{1}, Q_{2}^{2}} \otimes \rho_{Q_{1}^{1}, Q_{1}^{2}}\right.$ [by $\operatorname{LOP}\left(Q^{1 \stackrel{\leftrightarrow}{\rightarrow}} W \stackrel{\aleph \rightsquigarrow}{\leftarrow} Q^{2}\right)$ operations]. By the Remark 9, we then get that the $\operatorname{LOCC}\left(Q^{1}, Q^{2}\right)$ operation that reproduces $\Lambda$ is also a $\operatorname{LOP}\left(Q^{1 \stackrel{\leftrightarrow}{\rightarrow}} W \stackrel{\rightsquigarrow}{\longleftarrow} Q^{2}\right)$ operation, which concludes the proof of this direction.

For the converse, assume that a protocol is given, implementing $\Lambda$ by elemental operations of $\operatorname{LOP}\left(Q^{1 \stackrel{m}{\rightarrow}} W \stackrel{\rightsquigarrow}{\leftarrow} Q^{2}\right)$, using an ancillary state $\sum_{i j} r_{i, j}|i\rangle\left\langle\left. j\right|_{W}\right.$. To get the equivalent $\operatorname{LOCC}\left(Q^{1}, Q^{2}\right)$ protocol using the ancillary state $\sum_{i j} r_{i, j}|i i\rangle\left\langle\left. j j\right|_{Q_{2}^{1}, Q_{2}^{2}}\right.$, replace the elemental operations of the given protocol in the following way by $\operatorname{LOCC}\left(Q^{1}, Q^{2}\right)$ operations:

(1) Permutations: $\sum_{i}|\sigma(i)\rangle\left\langle\left. i\right|_{W}\right.$, by permutations on both sides: $\sum_{i}|\sigma(i)\rangle\left\langle\left. i\right|_{Q_{2}^{1}}\right.$ followed by $\left.\sum_{i} \mid \sigma(i)\right\rangle\left\langle\left. i\right|_{Q_{2}^{2} \text {. }}\right.$

(2) Phases: $\sum_{j} e^{i \phi(j)}|j\rangle\left\langle\left. j\right|_{W}\right.$, by phases on one side: $\sum_{j} e^{i \phi(j)}|j\rangle\left\langle\left. j\right|_{Q_{2}^{1}}\right.$

(3) Observed quantum operations: $|\alpha\rangle_{W_{a}} \otimes K_{Q^{s}}^{\alpha}$, by the same operation, with the outcome in an ancilla of the system $Q^{s}$, classically communicating the result $\alpha$ to the other side $Q^{\urcorner s}$ and encoding it there as well: $|\alpha\rangle_{Q_{a}^{s}} \otimes K_{Q^{s}}^{\alpha}$ followed by $|\alpha\rangle_{Q_{a}^{-s}}$.

(4) Classical to quantum forwarding: $\sum_{k}\left\langle\left. k\right|_{W_{a}} \otimes \mid k\right\rangle_{Q_{a}^{s}}$, by first deleting the copy on $Q_{2}^{\urcorner s}$ (by doing a Fourier measurement, followed by a correction of the phase on $Q_{2}^{s}$, as in the proof of Lemma 2), followed by the trivial forwarding: $\sum_{k}\left\langle\left. k\right|_{Q_{2}^{s}} \otimes \mid k\right\rangle_{Q_{a}^{s}}$.

Note that, by doing these replacements (and merging the ancillary Hilbert spaces with $Q_{2}^{j}, j=1,2$ after each step), a generic state $\sum_{i, j} s_{i, j}|i\rangle\left\langle\left. j\right|_{W} \otimes \tau(i, j)_{Q_{1}^{1}, Q_{1}^{2}}\right.$ in any step of the protocol gets mapped to $\sum_{i, j} s_{i, j}|i i\rangle\left\langle\left. j j\right|_{Q_{2}^{1}, Q_{2}^{2}} \otimes \tau(i, j)_{Q_{1}^{1}, Q_{1}^{2}}\right.$, from which the assertion follows.

A direct corollary of Remark 9 is Theorem 10, which introduces useful conditions for state transformations in multipartite entanglement.

Theorem (10). Let $\rho, \sigma$ be states on $W$. If $\exists \tau_{W}$, a state on $W$, s.t.

$$
\left.\tau_{W} \stackrel{\operatorname{LOP}\left(Q^{i(1)} \stackrel{\leftrightarrow}{\rightarrow}\right.}{\longrightarrow}{ }^{j(1)} \cdots\right)
$$

but

$$
\tau_{W} \stackrel{\operatorname{LOP}\left(Q^{i(1)} \stackrel{m}{\nrightarrow} W^{j(1)} \cdots\right)}{\rightarrow} \sigma
$$

then it follows that $\stackrel{\operatorname{LOCC}\left(Q^{1}, \ldots, Q^{n}\right)}{\rightarrow} \sigma$.
Proof.-Assume the theorem is not valid, that is,

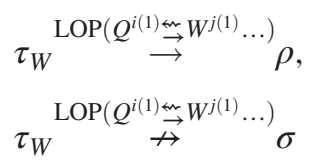

and

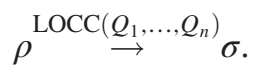

From Remark 9 (Point 2), it follows that

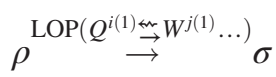

and, therefore,

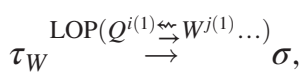

a contradiction.

\section{APPENDIX D: DIFFERENT TYPES OF ENTANGLEMENT}

Example 11.1. The least coherent state necessary to produce the $\left|G H Z_{n}\right\rangle_{Q^{1}, \ldots, Q^{n}}=(1 / \sqrt{2})\left(|0\rangle^{\otimes n}+|1\rangle^{\otimes n}\right)$ state by $\operatorname{LOP}\left(W \stackrel{\rightsquigarrow}{\leftarrow} Q^{1} \otimes \ldots Q^{n}\right) \quad$ is given by $|+\rangle_{W}=$ $(1 / \sqrt{2})(|0\rangle+|1\rangle)$. Similarly, one can produce $\left|\mathcal{W}_{n}\right\rangle_{Q^{1}, \ldots, Q^{n}}=(1 / \sqrt{n})(|0, \ldots 0,1\rangle+\ldots|1,0, \ldots, 0\rangle$ using $\operatorname{LOP}\left(W \stackrel{\rightsquigarrow}{\leftarrow} Q^{1} \otimes \ldots Q^{n}\right)$ from $\left|+_{\log _{2}(n)}\right\rangle_{W}=(1 / \sqrt{n})(|0\rangle+$ $\cdots+|n-1\rangle)$.

The least coherent state necessary to produce the $\left|G H Z_{n}\right\rangle_{Q^{1}, \ldots, Q^{n}} \quad$ state $\quad$ by $\operatorname{LOP}\left(Q^{1 \stackrel{\leftrightarrow}{\rightarrow}} W^{1} \stackrel{\leftrightarrow}{\leftarrow} Q^{2 \stackrel{\leftrightarrow}{\rightarrow}} \cdots\right.$ $\left.W^{n-1} \stackrel{\aleph}{\leftarrow} Q^{n}\right) \quad$ is given by $\left|{ }_{n-1}\right\rangle_{W^{1}, \ldots W^{n-1}}=$ $[(1 / \sqrt{2})(|0\rangle+|1\rangle)]^{\otimes(n-1)}$. Similarly, one can produce $\left|\mathcal{W}_{3}\right\rangle_{Q^{1}, Q^{2}, Q^{3}}$ by $|+\mathcal{W}\rangle_{W^{1}, W^{2}}:=(1 / \sqrt{2})(|0\rangle+|1\rangle) \otimes(1 / \sqrt{3}) \times$

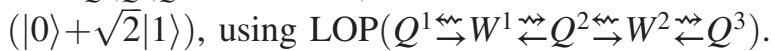

Proof.-We start by giving explicit protocols that do the conversions. For the $\left|G H Z_{n}\right\rangle_{Q^{1}, \ldots, Q^{n}}$ state, we simply apply a CNOT (a permutation) on $|+\rangle_{W_{1}} \otimes|0\rangle_{W_{2}, \ldots, W_{n-1}}^{(n-1)} n-1$ times (on $W_{1}, W_{j}$ ), resulting in $\left|G H Z_{n}\right\rangle_{W}$, and then we forward the respective subsystems. For the $|\mathcal{W}\rangle$ state, we apply a permutation that brings $|i\rangle_{W_{1}} \otimes|0\rangle_{W_{2}, \ldots, W_{n-1}}^{(n-1)}$ to $|0\rangle_{W_{1}} \ldots$ $|0\rangle|1\rangle_{W_{i}}|0\rangle \ldots|0\rangle_{W_{n-1}} \quad$ to $\quad\left|{ }_{\log _{2}(n)}\right\rangle_{W_{1}} \otimes|0\rangle_{W_{2}, \ldots, W_{n-1}}^{(n-1)}=$ $(1 / \sqrt{n})(|0\rangle+\cdots+|n-1\rangle) \otimes|0\rangle_{W_{2}, \ldots, W_{n-1}}^{(n-1)}$ and forward the subsystems to the different parties. That the generation of the $\left|G H Z_{n}\right\rangle$ state is optimal is simply seen by the fact that $\left|G H Z_{2}\right\rangle$ is the state with the minimal coherence rank [44] having the relative entropy of coherence [23] equal to the relative entropy of entanglement $[48,67,68]$ of $\left|G H Z_{n}\right\rangle_{Q^{1}, \ldots, Q^{n}}$, namely, 1. 
For the case with more than one wire, to prepare the $\left|G H Z_{n}\right\rangle_{Q^{1}, \ldots, Q^{n}}$ state from $\left|+_{n-1}\right\rangle_{W^{1}, \ldots, W^{n-1}}$, we do a local CNOT on each wire, after preparing an ancillary state $|0\rangle W_{2}^{j}$, effectively "doubling" the states, resulting in $\left|G H Z_{2}\right\rangle_{\left(W_{1}^{1}, W_{2}^{1}\right), \ldots,\left(W_{1}^{n-1}, W_{2}^{n-1}\right)}^{\otimes(n-1)}$. We then forward each half of the system of the respective wires to the quantum systems

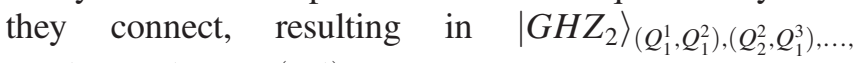
$Q_{1}^{n-1},\left(Q_{2}^{n-1}, Q_{1}^{n}\right)^{\otimes(n-1)}$. We can then double the system $Q_{1}^{2}$, resulting in the state $\left|G H Z_{3}\right\rangle_{Q_{1}^{1}, Q_{1}^{2}, Q_{3}^{2}}$. We can then use the $\left|G H Z_{2}\right\rangle_{\left(Q_{2}^{2}, Q_{1}^{3}\right)}$ to teleport the system $Q_{3}^{2}$ to $Q_{3}^{3}$, and so on. Iteratively we get the wanted $\left|G H Z_{n}\right\rangle_{Q^{1}, \ldots, Q^{n}}$ state. The optimality follows from any bipartitions being equivalent to $\left|G H Z_{2}\right\rangle$ states.

Starting from the $\left|+{ }_{\mathcal{W}}\right\rangle_{W^{1}, W^{2}}$ state, we first "double" each of the sides and then make a permutation $1 \leftrightarrow 0$ on the site $W_{2}^{2}$, which puts the system into the state $\left|G H Z_{2}\right\rangle_{W_{1}^{1}, W_{2}^{1}} \otimes(\sqrt{2} / \sqrt{3})(|0,1\rangle+(1 / \sqrt{3})|1,0\rangle)_{W_{1}^{2}, W_{2}^{2}}$. We continue by forwarding the system $W_{2}^{2}$ to $Q_{1}^{2}$. On this site $\left(Q^{2}\right)$, we then continue by applying the operation $|0,0\rangle_{Q_{1}^{2}, Q_{2}^{2}}$ $\left\langle\left. 0\right|_{Q_{1}^{2}}+(1 / \sqrt{2}) \mid 0,1\right\rangle_{Q_{1}^{2}, Q_{2}^{2}}\left\langle\left. 1\right|_{Q_{1}^{2}}+(1 / \sqrt{2}) \mid 1,0\right\rangle_{Q_{1}^{2}, Q_{2}^{2}}\left\langle\left. 1\right|_{Q_{1}^{2}}\right.$, leaving us with the $|\mathcal{W}\rangle$ state on $Q_{1}^{2} \otimes Q_{2}^{2} \otimes W_{1}^{2}$. The next step is to forward the system $W_{1}^{2}$ to $Q^{3}$. Finally, one can distribute the $\left|G H Z_{2}\right\rangle_{W_{1}^{1}, W_{2}^{1}}$ state to the connected quantum systems (yielding $\left|G H Z_{2}\right\rangle_{Q_{1}^{1}, Q_{3}^{2}}$ ) and use this to teleport via LOCC and, hence, LOP, the system $Q_{2}^{2}$ to $Q_{2}^{1}$. The protocol thus results in $|\mathcal{W}\rangle_{Q_{2}^{1}, Q_{1}^{2}, Q^{3}}$.

\section{APPENDIX E: ALL NOT INCOHERENT- QUANTUM STATES ARE RESOURCE STATES}

In this section, we show by a very simple (but highly inefficient) protocol that any state that does not have the form $\sum_{i}|i\rangle\left\langle\left. i\right|_{W} \otimes \rho(i)_{Q}\right.$ is maximally useful, in the sense that, having enough such states as ancillae, one can do any operation. If a state does not have this form, it must have the form $\sum_{i, j}|i\rangle\left\langle\left. j\right|_{W} \otimes \rho(i, j)_{Q}\right.$, with $\rho\left(i_{0}, j_{0}\right) \neq 0$ for some $i_{0}$, $j_{0}$ (without loss of generality $i_{0}=1, j_{0}=2$ ). The first step of the protocol is to double the state to get $\sum_{i, j}|i\rangle\left\langle\left. j\right|_{W} \otimes \mid i\right\rangle\left\langle\left. j\right|_{Q_{1}} \otimes \rho(i, j)_{Q_{2}}\right.$. To simplify the analysis we now note that we can make the measurement $K_{1}=1 / \sqrt{2}(\langle 1|+\langle 2|), K_{2}=\sqrt{1-K_{1}^{\dagger} K_{1}}$ on $Q_{1}$, which, with nonzero probability, will result in a state proportional to $\sum_{i, j \in\{1,2\}}|i\rangle\left\langle\left. j\right|_{W} \otimes \rho(i, j)_{Q_{2}}\right.$. That means that, as long as we are not interested in the optimal distillation protocol, we can start without loss of generality with the latter state. The next thing to note is that, for a state of this form, there is always a measurement on $Q_{2}$ that will yield some state with coherence on $W$ with nonzero probability (the algorithm is given in the proof of Theorem 2 in Ref. [28]). That means that we can start without loss of generality with a state $\sum_{i, j \in\{1,2\}}|i\rangle\left\langle\left. j\right|_{W} \sigma(i, j)\right.$, with $\sigma(1,2) \neq 0$. By a rotation, we get $\sigma(1,2)>0$, and, by the map that is given by the identity with probability $1 / 2$ and the permutation $1 \leftrightarrow 2$ with probability $1 / 2$, we can assume $\sigma(1,1)=\sigma(2,2)=1 / 2$. Having a state $\sigma_{1}(1,2)=p / 2$ on $W_{1}$ and adding a second state with $\sigma_{2}(1,2)=q / 2>0 W_{2}$, one can first do a CNOT with control $W_{1}$ acting on $W_{2}$, followed by the measurement $\left[\left\langle+\left.\right|_{W_{1}}=\left(\left\langle\left. 1\right|_{W_{1}}+\left\langle\left. 2\right|_{W_{1}}\right) / \sqrt{2}, \quad\left\langle-\left.\right|_{W_{1}}=\left(\left\langle\left. 1\right|_{W_{1}}-\right.\right.\right.\right.\right.\right.\right.$ $\left.\left\langle\left. 2\right|_{W_{1}}\right) / \sqrt{2}\right]$. With probability $\frac{1}{2}(1+p q)$, this yields the result "+" and the state

$$
\left(\begin{array}{cc}
\frac{1}{2} & \frac{p+q}{2 p q+2} \\
\frac{p+q}{2 p q+2} & \frac{1}{2}
\end{array}\right),
$$

and with probability $\frac{1}{2}(1-p q)$ one gets the result "_-" and the state

$$
\left(\begin{array}{cc}
\frac{1}{2} & \frac{p-q}{2-2 p q} \\
\frac{p-q}{2-2 p q} & \frac{1}{2}
\end{array}\right) .
$$

Notice that, by repeating the sequence many times (adding many times a state with coherence $q$ ), if one gets symmetric outcomes (the same number of + and - ), the resulting state is equal to the initial one, while the probability to get outcomes with more + is higher than that to get outcomes with more -. There is, therefore, a bias to get more coherent states over time. Also, the protocol only saturates at $p_{\text {final }}=1$. This means that, for a given $\epsilon$, having enough copies of any state that is not incoherent quantum, LOP allows us to prepare a maximally coherent state with fidelity $f>1-\epsilon$ and probability $p>1-\epsilon$. A simulation of this protocol is given in Fig. 7. Of course,

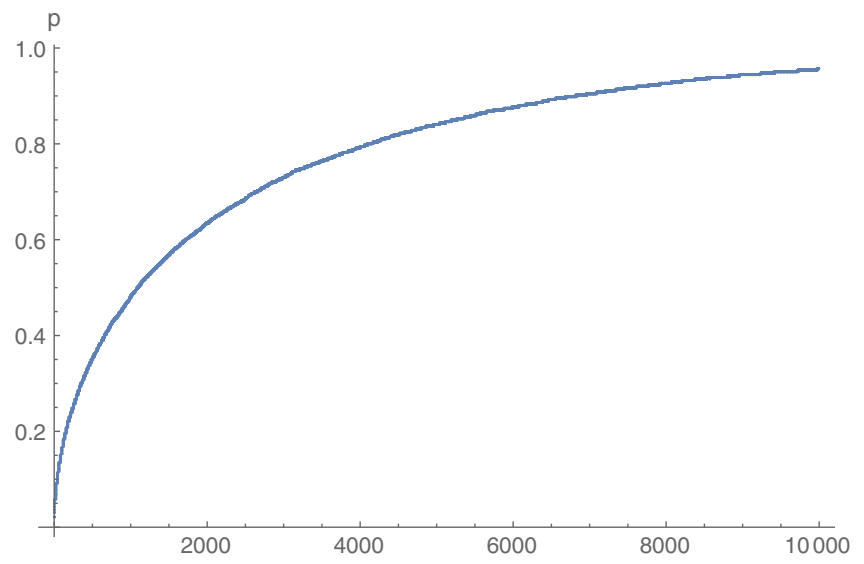

FIG. 7. The figure shows a simulation of the protocol discussed in Appendix E. Repeatedly, a state with 11-coherence 0.01 is used to increase the 11-coherence $p / 2$ of a given state (also initialized with coherence 0.01 ). If the coherence becomes negative, the state is dropped. This is repeated 10000 times to get the average behavior. One sees that, at some point, the protocol saturates with coherence $p / 2 \approx 0.5$. 
the protocol is suboptimal in many ways: It only considers two-dimensional coherence and destroys even a part of that by making the state symmetric at the beginning. Furthermore, one could maybe improve the algorithm by grouping states. In any case, it shows that one can distillate coherence in this setting, similar to how it was done in Ref. [24] using incoherent operations, even though the specific protocol given there does not seem to be easily adapted to LOP. It remains an interesting open question what is the best possible distillation rate of coherence in LOP.

\section{APPENDIX F: COUNTEREXAMPLE}

Lemma 12. Be A a rank 1 incoherent Kraus operator, and $\left\{\mathbf{B}_{s}\right\}$ a set of incoherent Kraus operators such that

$$
\mathbf{A}^{\dagger} \mathbf{A}=\sum_{s} \mathbf{B}_{s}^{\dagger} \mathbf{B}_{s} .
$$

Then, $\mathbf{B}_{s}=\lambda_{s} \mathbf{U}_{s} \mathbf{A}$ for certain $\lambda_{s}>0$ and $\mathbf{U}_{s}$ incoherent unitary operations.

Proof.-Since $\mathbf{A}^{\dagger} \mathbf{A}$ is rank 1, and $\mathbf{B}_{s}^{\dagger} \mathbf{B}_{s}$ are all positive, then $\mathbf{B}_{s}^{\dagger} \mathbf{B}_{s}=\lambda_{s}^{2} \mathbf{A}^{\dagger} \mathbf{A}$ for certain $\lambda_{s}$, such that $\sum_{s} \lambda_{s}^{2}=1$. Therefore, from the singular value decomposition theorem, $\mathbf{A}^{\dagger} \mathbf{A}=\left(\mathbf{B}_{s} / \lambda_{s}\right)^{\dagger}\left(\mathbf{B}_{s} / \lambda_{s}\right)$ iff $\mathbf{B}_{s} / \lambda_{s}=\mathbf{U}_{s} \mathbf{A}$ for certain unitaries $\mathbf{U}_{s}$. Finally, because $\mathbf{A}$ and $\mathbf{B}_{s}$ are incoherent, $\mathbf{U}_{s}$ must be incoherent.

\section{Lemma: General form for three-level systems}

From Proposition 3, the most general form of an operation $\quad \Lambda \in \operatorname{LOP}(W \stackrel{\rightsquigarrow}{\leftarrow} Q)$, with $\quad \operatorname{dim}(W)=3$, $\operatorname{dim}(Q)=1$, is given by

$$
\begin{aligned}
\Lambda(\rho)= & \sum_{\alpha_{1} \in I_{1,3}} \mathbf{F}_{1}^{\alpha_{1}} \rho \mathbf{F}_{1}^{\alpha_{1} \dagger}+\sum_{\alpha_{2} \in I_{2,2 \alpha_{1} \in I_{1,2}}} \mathbf{F}_{2,2}^{\alpha_{2} \alpha_{1}} \rho \mathbf{F}_{2,2}^{\alpha_{2} \alpha_{1} \dagger} \\
& +\sum_{\alpha_{2} \in I_{2,1} \alpha_{1} \in I_{1,1}} \mathbf{F}_{2,1}^{\alpha_{2} \alpha_{1}} \rho \mathbf{F}_{2,1}^{\alpha_{2} \alpha_{1} \dagger} \\
& +\sum_{\alpha_{3} \in I_{3,1} \alpha_{2} \in I_{2,2}^{\prime} \alpha_{1} \in I_{1,2}} \mathbf{F}_{3}^{\alpha_{3} \alpha_{2} \alpha_{1}} \rho \mathbf{F}_{3}^{\alpha_{3} \alpha_{2} \alpha_{1} \dagger}
\end{aligned}
$$

with [defining for notational ease $c u t_{t}(x):=\min (t, x)$ ]

$$
\begin{aligned}
\mathbf{F}_{1}^{\alpha_{1}} & =\sum_{m} q_{m}^{\alpha_{1}}\left|\sigma^{\alpha_{1}}(m)\right\rangle\langle m| \\
\mathbf{F}_{2,2}^{\alpha_{2} \alpha_{1}} & =\sum_{m} q_{m}^{\alpha_{2} \alpha_{1}}\left|\left(\sigma^{\alpha_{2} \alpha_{1}} \circ \mathrm{cut}_{2} \circ \sigma^{\alpha_{1}}\right)(m)\right\rangle\langle m| \\
\mathbf{F}_{2,1}^{\alpha_{2} \alpha_{1}} & =\sum_{m} q_{m}^{\alpha_{2} \alpha_{1}}\left|\left(\sigma^{\alpha_{2} \alpha_{1}} \circ \mathrm{cut}_{1} \circ \sigma^{\alpha_{1}}\right)(m)\right\rangle\langle m| \\
\mathbf{F}_{3}^{\alpha_{3} \alpha_{2} \alpha_{1}} & =\sum_{m} q_{m}^{\alpha_{3} \alpha_{2} \alpha_{1}}\left|\left(\sigma^{\alpha_{3} \alpha_{2} \alpha_{1}} \circ \mathrm{cut}_{1} \circ \sigma^{\alpha_{2} \alpha_{1}} \circ \mathrm{cut}_{2} \circ \sigma^{\alpha_{1}}\right)(m)\right\rangle\langle m|
\end{aligned}
$$

and $\quad \alpha_{1} \in I_{1,3} \cup I_{1,2} \cup I_{1,1}, \quad \alpha_{2} \in I_{2,1} \cup I_{2,2} \cup I_{2,2}^{\prime}$, and $\alpha_{3} \in I_{3,1}$. Because of the trace preserving condition, these operators must satisfy the constraints

$$
\begin{array}{ll}
\sum_{\alpha_{2} \in I_{2,1}} \mathbf{F}_{2,1}^{\alpha_{2} \alpha_{1} \dagger} \mathbf{F}_{2,1}^{\alpha_{2} \alpha_{1}}=\mathbf{F}_{1}^{\alpha_{1} \dagger} \mathbf{F}_{1}^{\alpha_{1}}, & \alpha_{1} \in I_{1,1} \\
\sum_{\alpha_{2} \in I_{2,2}} \mathbf{F}_{2,2}^{\alpha_{2} \alpha_{1} \dagger} \mathbf{F}_{2,2}^{\alpha_{2} \alpha_{1}}=\mathbf{F}_{1}^{\alpha_{1} \dagger} \mathbf{F}_{1}^{\alpha_{1}}, & \alpha_{1} \in I_{1,2}
\end{array}
$$

$$
\begin{aligned}
\sum_{\alpha_{3} \in I_{3,1}} \mathbf{F}_{3}^{\alpha_{3} \alpha_{2} \alpha_{1} \dagger} \mathbf{F}_{3}^{\alpha_{3} \alpha_{2} \alpha_{1}} & =\mathbf{F}_{2,2}^{\alpha_{2} \alpha_{1} \dagger} \mathbf{F}_{2,2}^{\alpha_{2} \alpha_{1}}, \\
\alpha_{1} & \in I_{1,2}, \quad \alpha_{2} \in I_{2,2}^{\prime}
\end{aligned}
$$

$$
\sum_{\alpha_{2} \in I_{2,2} \cup I_{2,2}^{\prime}} \mathbf{F}_{2,2}^{\alpha_{2} \alpha_{1} \dagger} \mathbf{F}_{2,2}^{\alpha_{2} \alpha_{1}}=\mathbf{F}_{1}^{\alpha_{1} \dagger} \mathbf{F}_{1}^{\alpha_{1}}, \quad \alpha_{1} \in I_{1,2}
$$

$\sum_{\alpha_{1} \in I_{1,1} \cup I_{1,2} \cup I_{1,3}} \mathbf{F}_{1}^{\alpha_{1} \dagger} \mathbf{F}_{1}^{\alpha_{1}}=\mathbf{1}$.

Proof.-It follows from Proposition 3, if we assume, without loss of generality, that the initial global state is a product of the initial state $\rho$ on $W$, and reference ancillary state $\rho_{Q}=|0\rangle\left\langle\left. 0\right|_{Q}\right.$, then the final state is given by

$$
\begin{aligned}
\Lambda(\rho) & =\operatorname{Tr}_{Q} \Lambda_{W Q}\left(\rho \otimes|0\rangle\left\langle\left. 0\right|_{Q}\right)\right. \\
& =\sum_{\vec{\alpha}} \sum_{m, m^{\prime}} Q_{m m^{\prime}}^{\vec{\alpha}}\left|f^{\vec{\alpha}}(m)\right\rangle\left\langle m|\rho| m^{\prime}\right\rangle\left\langle f^{\vec{\alpha}}\left(m^{\prime}\right)\right|
\end{aligned}
$$

where $f^{\vec{\alpha}}=\sigma^{\alpha_{k}} \circ c u t^{r_{\alpha_{k}} \ldots \alpha_{1}} \circ \ldots \circ \sigma^{\alpha_{1}}$ and $Q_{m m^{\prime}}^{\vec{\alpha}}=\operatorname{Tr}\left(\mathbf{E}_{m}^{\vec{\alpha}}|0\rangle \times\right.$ $\left.\langle 0| \mathbf{E}_{m^{\prime}}^{\overrightarrow{\alpha^{\dagger}}}\right)=\left\langle 0\left|\mathbf{E}_{m^{\prime}}^{\vec{\alpha} \dagger} \mathbf{E}_{m}^{\vec{\alpha}}\right| 0\right\rangle=Q_{m m^{\prime}}^{\vec{\alpha} \dagger}$, with $\mathbf{E}_{m}^{\vec{\alpha}}=\mathbf{E}_{f^{\alpha_{k} \ldots \alpha_{1}}(m)}^{\alpha_{k} \ldots \alpha_{1}} \ldots$ $\mathbf{E}_{f^{\alpha_{1}(m)}}^{\alpha_{2} \alpha_{1}} \mathbf{E}_{m}^{\alpha_{1}}$ the sequence of conditional operators applied at each step. By construction, $Q_{m m^{\prime}}^{\vec{\alpha}}$ is a Grahm matrix, and hence, it is positive semidefinite. With $\zeta_{m}^{\vec{\alpha} \lambda}=\left\langle\lambda\left|\mathbf{E}_{m}^{\vec{\alpha}}\right| 0\right\rangle$, it can be expended as $Q_{m m^{\prime}}^{\alpha}=\sum_{\lambda} \zeta_{m}^{\vec{\alpha} \lambda} \zeta_{m^{\prime}}^{\vec{\alpha} \lambda}$, and we see that the general form of $\Lambda$ is given by

$$
\Lambda(\rho)=\sum_{\vec{\alpha}, \lambda} \tilde{\mathbf{F}}^{\vec{\alpha} \lambda} \rho \tilde{\mathbf{F}}^{\vec{\alpha} \lambda \dagger}
$$

with $\tilde{\mathbf{F}}^{\vec{\alpha} \lambda}=\sum_{m} \zeta_{m}^{\vec{\alpha} \lambda}\left|f_{m}^{\vec{\alpha}}\right\rangle\langle m|$. The $\lambda$ coefficient in the sum can be assimilated to the last set of outcomes, leading to the form presented in the theorem.

\section{Proposition: $\mathrm{LOP} \neq \mathrm{IQO}$}

Suppose now that the incoherent operation $\Lambda(\rho)=$ $\sum_{s=1}^{4} \mathbf{K}_{s} \rho \mathbf{K}_{s}^{\dagger}$, defined by 


$$
\begin{aligned}
\mathbf{K}_{1}=\left(\begin{array}{ccc}
\frac{1}{2} & -\frac{1}{2} & 0 \\
0 & 0 & \frac{1}{2} \\
0 & 0 & 0
\end{array}\right) & \mathbf{K}_{2}=\left(\begin{array}{ccc}
\frac{1}{2} & 0 & -\frac{1}{2} \\
0 & \frac{1}{2} & 0 \\
0 & 0 & 0
\end{array}\right) \\
\mathbf{K}_{3}=\left(\begin{array}{ccc}
0 & \frac{1}{2} & -\frac{1}{2} \\
\frac{1}{2} & 0 & 00 \\
0 & 0 & 0
\end{array}\right) & \mathbf{K}_{4}=\left(\begin{array}{ccc}
\frac{1}{2} & \frac{1}{2} & \frac{1}{2} \\
0 & 0 & 0 \\
0 & 0 & 0
\end{array}\right),
\end{aligned}
$$

admits that decomposition. Since $\sum_{\mu} \lambda_{\mu} \mathbf{K}_{s_{\mu}}$ is an incoherent Kraus operator iff $s_{\mu}=$ const, it follows that, in any possible incoherent Kraus representation of $\Lambda(\rho)$, all the Kraus operators need to be proportional to some $\mathbf{K}_{s}$, hence,

$$
\begin{aligned}
\mathbf{F}_{1}^{\alpha_{1}} & =0 \quad \forall \alpha_{1} \in I_{1,3} \\
\mathbf{F}_{2,2}^{\alpha_{2} \alpha_{1}} & =\zeta_{2,2}^{\alpha_{2}, \alpha_{1}} \mathbf{K}_{m_{\alpha_{2}, \alpha_{1}}} \quad m_{\alpha_{2}, \alpha_{1}} \in\{1,2,3\} \\
\mathbf{F}_{2,1}^{\alpha_{2} \alpha_{1}} & =\zeta_{2,1}^{\alpha_{2}, \alpha_{1}} \mathbf{K}_{4} \\
\mathbf{F}_{3}^{\alpha_{3} \alpha_{2} \alpha_{1}} & =\zeta_{3}^{\alpha_{3}, \alpha_{2}, \alpha_{1}} \mathbf{K}_{4} .
\end{aligned}
$$

Plugging this into the trace preserving conditions F3 results in

$$
\begin{gathered}
\mathbf{K}_{4}^{\dagger} \mathbf{K}_{4}\left(\sum_{\alpha_{3} \in I_{3,1}}\left|\zeta_{3}^{\alpha_{3} \alpha_{2} \alpha_{1}}\right|^{2}\right)=\mathbf{F}_{2,2}^{\alpha_{2} \alpha_{1} \dagger} \mathbf{F}_{2,2}^{\alpha_{2} \alpha_{1}} \\
\mathbf{K}_{4}^{\dagger} \mathbf{K}_{4}\left(\sum_{\alpha_{2,1}}\left|\zeta_{2,1}^{\alpha_{2} \alpha_{1}}\right|^{2}\right)=\mathbf{F}_{1}^{\alpha_{1} \dagger} \mathbf{F}_{1}^{\alpha_{1}} .
\end{gathered}
$$

If the left-hand side is nonzero in the first (second) condition, it implies that, for certain $\alpha_{1}, \alpha_{2}\left(\alpha_{1}\right), \mathbf{F}_{2,2}^{\alpha_{2} \alpha_{1}}$ $\left(\mathbf{F}_{1}^{\alpha_{1}}\right)$ should (by Lemma 12) be proportional to $\mathbf{U K}_{4}$ for certain $\mathbf{U}$ unitary incoherent, but then, against the hypothesis, $\mathbf{F}_{2,2}^{\alpha_{2} \alpha_{1}}\left(\mathbf{F}_{1}^{\alpha_{1}}\right)$ needs to be of the form of Eq. (F2b) [(F2a)]. On the other hand, if both expressions are zero, $\mathbf{K}_{4}$ cannot appear in the Kraus decomposition of $\Lambda$, leading to a contradiction. Therefore, the explicitly incoherent operation $\Lambda$ is not in $\operatorname{LOP}(W \rightsquigarrow Q)$, while being an element of IO and, hence, also of GOIA and IQO. One can easily check that $\Lambda$ is also an element of DIO.

[1] E. Schrödinger, Die Gegenwärtige Situation in der Quantenmechanik, Naturwissenschaften 23, 807 (1935) [, The Present Situation in Quantum Mechanics: A Translation of Schrödinger's "Cat Paradox" Paper, Proc. Am. Philos. Soc. 124, 323 (1980)].

[2] A. Einstein, B. Podolsky, and N. Rosen, Can QuantumMechanical Description of Physical Reality Be Considered Complete?, Phys. Rev. 47, 777 (1935).

[3] J. S. Bell, On the Problem of Hidden Variables in Quantum Mechanics, Rev. Mod. Phys. 38, 447 (1966).
[4] A. Aspect, P. Grangier, and G. Roger, Experimental Tests of Realistic Local Theories via Bell's Theorem, Phys. Rev. Lett. 47, 460 (1981).

[5] B. Hensen, H. Bernien, A. E. Dreau, A. Reiserer, N. Kalb, M. S. Blok, J. Ruitenberg, R. F. L. Vermeulen, R. N. Schouten, C. Abellan, W. Amaya, V. Pruneri, M. W. Mitchell, M. Markham, D. J. Twitchen, D. Elkouss, S. Wehner, T. H. Taminiau, and R. Hanson, Loophole-Free Bell Inequality Violation Using Electron Spins Separated by 1.3 Kilometres, Nature (London) 526, 682 (2015).

[6] J. F. Clauser, M. A. Horne, A. Shimony, and R. A. Holt, Proposed Experiment to Test Local Hidden-Variable Theories, Phys. Rev. Lett. 23, 880 (1969).

[7] S. Kochen and E. P. Specker, The Problem of Hidden Variables in Quantum Mechanics, in The Logico-Algebraic Approach to Quantum Mechanics: Volume I: Historical Evolution, edited by C. A. Hooker (Springer Netherlands, Dordrecht, 1975), pp. 293-328.

[8] N. D. Mermin, Hidden Variables and the Two Theorems of John Bell, Rev. Mod. Phys. 65, 803 (1993).

[9] E. Amselem, M. Rådmark, M. Bourennane, and A. Cabello, State-Independent Quantum Contextuality with Single Photons, Phys. Rev. Lett. 103, 160405 (2009).

[10] S. F. Huelga and M. B. Plenio, Vibrations, Quanta and Biology, Contemp. Phys. 54, 181 (2013).

[11] M. B. Plenio and S. Virmani, An Introduction to Entanglement Measures, Quantum Inf. Comput. 7, 1 (2007).

[12] R. Horodecki, P. Horodecki, M. Horodecki, and K. Horodecki, Quantum Entanglement, Rev. Mod. Phys. 81, 865 (2009).

[13] S. Parker and M. B. Plenio, Entanglement Simulations of Shor's Algorithm, J. Mod. Opt. 49, 1325 (2002).

[14] A. Datta, S. T. Flammia, and C. M. Caves, Entanglement and the Power of One Qubit, Phys. Rev. A 72, 042316 (2005).

[15] A. Datta, A. Shaji, and C. M. Caves, Quantum Discord and the Power of One Qubit, Phys. Rev. Lett. 100, 050502 (2008).

[16] B. P. Lanyon, M. Barbieri, M. P. Almeida, and A. G. White, Experimental Quantum Computing without Entanglement, Phys. Rev. Lett. 101, 200501 (2008).

[17] M. Ali, Quantum Dissonance and Deterministic Quantum Computation with a Single Qubit, Int. J. Quantum. Inform. 12, 1450037 (2014).

[18] H. Ollivier and W. H. Zurek, Quantum Discord: A Measure of the Quantumness of Correlations, Phys. Rev. Lett. 88, 017901 (2001).

[19] L. Henderson and V. Vedral, Classical, Quantum and Total Correlations, J. Phys. A 34, 6899 (2001).

[20] K. Modi, A. Brodutch, H. Cable, T. Paterek, and V. Vedral, The Classical-Quantum Boundary for Correlations: Discord and Related Measures, Rev. Mod. Phys. 84, 1655 (2012).

[21] G. Adesso, T. R. Bromley, and M. Cianciaruso, Measures and Applications of Quantum Correlations, J. Phys. A 49, 473001 (2016).

[22] J. Åberg, Quantifying Superposition, arXiv:quant-ph/ 0612146.

[23] T. Baumgratz, M. Cramer, and M. B. Plenio, Quantifying Coherence, Phys. Rev. Lett. 113, 140401 (2014).

[24] A. Winter and D. Yang, Operational Resource Theory of Coherence, Phys. Rev. Lett. 116, 120404 (2016). 
[25] A. Streltsov, G. Adesso, and M. B. Plenio, Colloquium: Quantum Coherence as a Resource, Rev. Mod. Phys. 89, 041003 (2017).

[26] A. Streltsov, U. Singh, H. S. Dhar, M. N. Bera, and G. Adesso, Measuring Quantum Coherence with Entanglement, Phys. Rev. Lett. 115, 020403 (2015).

[27] Y. Yao, X. Xiao, L. Ge, and C. P. Sun, Quantum Coherence in Multipartite Systems, Phys. Rev. A 92, 022112 (2015).

[28] E. Chitambar, A. Streltsov, S. Rana, M. N. Bera, G. Adesso, and M. Lewenstein, Assisted Distillation of Quantum Coherence, Phys. Rev. Lett. 116, 070402 (2016).

[29] E. Chitambar and M.-H. Hsieh, Relating the Resource Theories of Entanglement and Quantum Coherence, Phys. Rev. Lett. 117, 020402 (2016).

[30] A. Streltsov, E. Chitambar, S. Rana, M. N. Bera, A. Winter, and M. Lewenstein, Entanglement and Coherence in Quantum State Merging, Phys. Rev. Lett. 116, 240405 (2016).

[31] X. Hu, A. Milne, B. Zhang, and H. Fan, Quantum Coherence of Steered States, Sci. Rep. 6, 19365 (2016).

[32] X. Hu and H. Fan, Extracting Quantum Coherence via Steering, Sci. Rep. 6, 34380 (2016).

[33] M.-L. Hu and H. Fan, Relative Quantum Coherence, Incompatibility, and Quantum Correlations of States, Phys. Rev. A 95, 052106 (2017).

[34] H. Zhu, Z. Ma, Z. Cao, S.-M. Fei, and V. Vedral, Operational One-to-One Mapping between Coherence and Entanglement Measures, Phys. Rev. A 96, 032316 (2017).

[35] C. Radhakrishnan, M. Parthasarathy, S. Jambulingam, and T. Byrnes, Distribution of Quantum Coherence in Multipartite Systems, Phys. Rev. Lett. 116, 150504 (2016).

[36] A. Streltsov, S. Rana, M. N. Bera, and M. Lewenstein, Towards Resource Theory of Coherence in Distributed Scenarios, Phys. Rev. X 7, 011024 (2017).

[37] D. Girolami and B. Yadin, Witnessing Multipartite Entanglement by Detecting Asymmetry, Entropy 19, 124 (2017).

[38] K. Bu, L. Li, A. K. Pati, S.-M. Fei, and J. Wu, Distribution of Coherence in Multipartite Systems under Entropic Coherence Measure, arXiv:1710.08517v1.

[39] B. Yadin, J. Ma, D. Girolami, M. Gu, and V. Vedral, Quantum Processes Which Do Not Use Coherence, Phys. Rev. X 6, 041028 (2016).

[40] E. Chitambar and G. Gour, Critical Examination of Incoherent Operations and a Physically Consistent Resource Theory of Quantum Coherence, Phys. Rev. Lett. 117, 030401 (2016).

[41] I. Marvian and R. W. Spekkens, How to Quantify Coherence: Distinguishing Speakable and Unspeakable Notions, Phys. Rev. A 94, 052324 (2016).

[42] J. Ma, B. Yadin, D. Girolami, V. Vedral, and M. Gu, Converting Coherence to Quantum Correlations, Phys. Rev. Lett. 116, 160407 (2016).

[43] J. M. Matera, D. Egloff, N. Killoran, and M. B. Plenio, Coherent Control of Quantum Systems as a Resource Theory, Quantum Sci. Technol. 1, 01LT01 (2016).

[44] N. Killoran, F. E. S. Steinhoff, and M. B. Plenio, Converting Nonclassicality into Entanglement, Phys. Rev. Lett. 116, 080402 (2016).

[45] T. Theurer, N. Killoran, D. Egloff, and M. B. Plenio, Resource Theory of Superposition, Phys. Rev. Lett. 119, 230401 (2017).
[46] B. Regula, M. Piani, M. Cianciaruso, T. R. Bromley, A. Streltsov, and G. Adesso, Converting Multilevel Nonclassicality into Genuine Multipartite Entanglement, New J. Phys. 20, 033012 (2018).

[47] J. Sperling, E. Agudelo, I. A. Walmsley, and W. Vogel, Quantum Correlations in Composite Systems, J. Phys. B 50, 134003 (2017).

[48] V. Vedral, M. B. Plenio, M. A. Rippin, and P. L. Knight, Quantifying Entanglement, Phys. Rev. Lett. 78, 2275 (1997).

[49] S. Meznaric, S. R. Clark, and A. Datta, Quantifying the Nonclassicality of Operations, Phys. Rev. Lett. 110, 070502 (2013).

[50] Z.-W. Liu, X. Hu, and S. Lloyd, Resource Destroying Maps, Phys. Rev. Lett. 118, 060502 (2017).

[51] G. Gour and R. W. Spekkens, The Resource Theory of Quantum Reference Frames: Manipulations and Monotones, New J. Phys. 10, 033023 (2008).

[52] P. W. Shor and J. Preskill, Simple Proof of Security of the BB84 Quantum Key Distribution Protocol, Phys. Rev. Lett. 85, 441 (2000).

[53] K. Horodecki, M. Horodecki, P. Horodecki, and J. Oppenheim, Secure Key from Bound Entanglement, Phys. Rev. Lett. 94, 160502 (2005).

[54] T. S. Cubitt, F. Verstraete, W. Dür, and J. I. Cirac, Separable States Can Be Used to Distribute Entanglement, Phys. Rev. Lett. 91, 037902 (2003).

[55] W. Dür, G. Vidal, and J. I. Cirac, Three Qubits Can Be Entangled in Two Inequivalent Ways, Phys. Rev. A 62, 062314 (2000).

[56] M. Walter, D. Gross, and J. Eisert, Multi-partite Entanglement, arXiv:1612.02437v2.

[57] L. Lamata, J. León, D. Salgado, and E. Solano, Inductive Entanglement Classification of Four Qubits under Stochastic Local Operations and Classical Communication, Phys. Rev. A 75, 022318 (2007).

[58] M. Backens, Number of Superclasses of Four-Qubit Entangled States under the Inductive Entanglement Classification, Phys. Rev. A 95, 022329 (2017).

[59] J. I. de Vicente, T. Carle, C. Streitberger, and B. Kraus, Complete Set of Operational Measures for the Characterization of Three-Qubit Entanglement, Phys. Rev. Lett. 108, 060501 (2012).

[60] B. Regula, S. Di Martino, S. Lee, and G. Adesso, Strong Monogamy Conjecture for Multiqubit Entanglement: The Four-Qubit Case, Phys. Rev. Lett. 113, 110501 (2014).

[61] C. H. Bennett and G. Brassard, Quantum Cryptography: Public Key Distribution and Coin Tossing, in Proceedings of IEEE International Conference on Computers, Systems and Signal Processing, Bangalore, India, Dec. 10-12, 1984 (1984).

[62] For any ancilla $W_{2}$ to $W_{1}$, we fix an incoherent basis $\left(|j\rangle W_{1}\right.$, $|k\rangle_{W_{2}}$ ) and label the full basis of the new $W$ by natural numbers $|i\rangle_{W}=|i(j, k)\rangle_{W}$. We use implicitly the change of representation $|i(j, k)\rangle_{W} \equiv|j\rangle_{W_{1}} \otimes|k\rangle_{W_{2}}$. We also use the identification $c \otimes|\Psi\rangle=c|\Psi\rangle$ for scalars $c \in \mathbb{C}$ throughout and, similarly, $\left\langle\left. j\right|_{W_{s}} \otimes \mid j\right\rangle_{Q_{t}}=|j\rangle_{Q_{t}}\left\langle\left. j\right|_{W_{s}}\right.$.

[63] A. Streltsov, S. Rana, P. Boes, and J. Eisert, Structure of the Resource Theory of Quantum Coherence, Phys. Rev. Lett. 119, 140402 (2017). 
[64] E. Chitambar, Local Quantum Transformations Requiring Infinite Rounds of Classical Communication, Phys. Rev. Lett. 107, 190502 (2011).

[65] M. A. Nielsen and I. L. Chuang, Quantum Computation and Quantum Information 1st ed. (Cambridge University Press, Cambridge, England, 2000).

[66] Y. Huang, Computing Quantum Discord Is NP-Complete, New J. Phys. 16, 033027 (2014).

[67] M. B. Plenio and V. Vedral, Bounds on Relative Entropy of Entanglement for Multi-party Systems, J. Phys. A 34, 6997 (2001).

[68] T.-C. Wei, Relative Entropy of Entanglement for Multipartite Mixed States: Permutation-Invariant States and Dür States, Phys. Rev. A 78, 012327 (2008).

[69] F. Shahandeh, A. P. Lund, and T. C. Ralph, Quantum Correlations and Global Coherence in Distributed Quantum Computing, arXiv:1706.00478v2.

[70] J. I. de Vicente and A. Streltsov, Genuine Quantum Coherence, J. Phys. A 50, 045301 (2017).

[71] T. Biswas, M. García Díaz, and A. Winter, Interferometric Visibility and Coherence, Proc. R. Soc. A 473, 20170170 (2017).
[72] C. Weedbrook, S. Pirandola, R. García-Patrón, N. J. Cerf, T. C. Ralph, J. H. Shapiro, and S. Lloyd, Gaussian Quantum Information, Rev. Mod. Phys. 84, 621 (2012).

[73] L. Roa, A. Delgado, and I. Fuentes-Guridi, Optimal Conclusive Teleportation of Quantum States, Phys. Rev. A 68 , 022310 (2003).

[74] A. Smirne, D. Egloff, M. G. Díaz, M. B. Plenio, and S. F. Huelga, Coherence and Non-classicality of Quantum Markov Processes, arXiv:1709.05267v1.

[75] D. Janzing, P. Wocjan, R. Zeier, R. Geiss, and T. Beth, Thermodynamic Cost of Reliability and Low Temperatures: Tightening Landauer's Principle and the Second Law, Int. J. Theor. Phys. 39, 2717 (2000).

[76] M. Lostaglio, D. Jennings, and T. Rudolph, Description of Quantum Coherence in Thermodynamic Processes Requires Constraints Beyond Free Energy, Nat. Commun. 6, 6383 (2015).

[77] C. H. Bennett, D. P. DiVincenzo, C. A. Fuchs, T. Mor, E. Rains, P. W. Shor, J. A. Smolin, and W. K. Wootters, Quantum Nonlocality without Entanglement, Phys. Rev. A 59, 1070 (1999). 